\title{
Genome-wide identification of the amino acid permease genes and molecular characterization of their transcriptional responses to various nutrient stresses in allotetraploid rapeseed
}

Ting Zhou ${ }^{1 \dagger}$, Cai-peng Yue ${ }^{1 \dagger}$, Jin-yong Huang ${ }^{1}$, Jia-qian Cui ${ }^{1}$, Ying Liu', Wen-ming Wang ${ }^{1}$, Chuang Tian $^{2}$ and Ying-peng Hua ${ }^{1 *}$

\begin{abstract}
Background: Nitrogen $(\mathrm{N})$, referred to as a "life element", is a macronutrient essential for optimal plant growth and yield production. Amino acid (AA) permease (AAP) genes play pivotal roles in root import, long-distance translocation, remobilization of organic amide-N from source organs to sinks, and other environmental stress responses. However, few systematic analyses of AAPs have been reported in Brassica napus so far.

Results: In this study, we identified a total of 34 full-length AAP genes representing eight subgroups (AAP1-8) from the allotetraploid rapeseed genome $\left(A_{n} A_{n} C_{n} C_{n}, 2 n=4 x=38\right)$. Great differences in the homolog number among the BnaAAP subgroups might indicate their significant differential roles in the growth and development of rapeseed plants. The BnaAAPs were phylogenetically divided into three evolutionary clades, and the members in the same subgroups had similar physiochemical characteristics, gene/protein structures, and conserved AA transport motifs. Darwin's evolutionary analysis suggested that BnaAAPs were subjected to strong purifying selection pressure. Ciselement analysis showed potential differential transcriptional regulation of AAPs between the model Arabidopsis and B. napus. Differential expression of BnaAAPs under nitrate limitation, ammonium excess, phosphate shortage, boron deficiency, cadmium toxicity, and salt stress conditions indicated their potential involvement in diverse nutrient stress responses.

Conclusions: The genome-wide identification of BnaAAPs will provide a comprehensive insight into their family evolution and AAP-mediated AA transport under diverse abiotic stresses. The molecular characterization of core AAPs can provide elite gene resources and contribute to the genetic improvement of crop stress resistance through the modulation of AA transport.
\end{abstract}

Keywords: Allotetraploid, Amino acid permease, Brassica napus, Genome-wide identification, Nutrient stresses

\footnotetext{
* Correspondence: yingpenghua@zzu.edu.cn

${ }^{\dagger}$ Ting Zhou and Cai-peng Yue contributed equally to this work.

${ }^{1}$ School of Agricultural Sciences, Zhengzhou University, Zhengzhou 450000,

China

Full list of author information is available at the end of the article
}

(c) The Author(s). 2020 Open Access This article is licensed under a Creative Commons Attribution 4.0 International License, which permits use, sharing, adaptation, distribution and reproduction in any medium or format, as long as you give appropriate credit to the original author(s) and the source, provide a link to the Creative Commons licence, and indicate if changes were made. The images or other third party material in this article are included in the article's Creative Commons licence, unless indicated otherwise in a credit line to the material. If material is not included in the article's Creative Commons licence and your intended use is not permitted by statutory regulation or exceeds the permitted use, you will need to obtain permission directly from the copyright holder. To view a copy of this licence, visit http://creativecommons.org/licenses/by/4.0/ The Creative Commons Public Domain Dedication waiver (http://creativecommons.org/publicdomain/zero/1.0/) applies to the data made available in this article, unless otherwise stated in a credit line to the data. 


\section{Background}

Seed yield and protein content are largely reliant on abundant accumulation of nitrogen $(\mathrm{N})$, which is a macronutrient indispensable for optimal plant growth and organ development [1]. Plants absorb mainly inorganic $\mathrm{N}$ nutrients in the form of nitrate $\left(\mathrm{NO}_{3}{ }^{-}\right)$and ammonium $\left(\mathrm{NH}_{4}{ }^{+}\right)$, some of which are assimilated into amino acids (AAs) directly in the roots or were translocated to the shoots. In addition, plants can also directly transport AAs and other organic $\mathrm{N}$ compounds into roots in soils [2]. The development and metabolism of vegetative and reproductive organs require large numbers of AAs in the phloem. AAs derived from senescent leaves are the major $\mathrm{N}$ forms for seed $\mathrm{N}$ nutrients [3]. Therefore, efficient uptake and translocation of AAs are favorable for yield production, and also favorable for plant resistance against various stresses [4-7].

More than 100 putative AA transporters (AATs), including the Amino acid-Polyamine-Choline (APC) transporter superfamily and the Usually Multiple Acids Move In and Out Transporters (UmamiT) family, have been characterized in the model Arabidopsis. The APC family can be further grouped into three categories: Amino Acid Permeases (AAPs), Lysine/Histidine-like Transporters (LHTs) and Proline, and Glycine Betaine Transporters (ProTs) [8-10]. Among these AATs, AAPs are considered to be a moderate-affinity system with broad substrate specificity. In plants, AAPs are also involved in various physiological processes, including AA uptake [11], phloem loading or xylem-phloem transfer [12], seed loading [13], and grain yield [14]. AAPs localized on the plasma membrane are involved in cellular $\mathrm{H}^{+}$-coupled intake of a broad range of AAs. A recent study has reported that the genetic manipulation of AAPs improves AA transport from sources to sinks, which further enhances plant $\mathrm{N}$ use efficiency (NUE) [15]. Each member of the $A A P$ family shows a specific temporal and spatial expression pattern, which indicates the non-redundant roles of $A A P s$ in plants [11].

In Arabidopsis, the AAP family contains eight protein members (AtAAP1-8) that generally transport neutral and acidic AAs with moderate affinity except that AtAAP3 and AtAAP5 also transport basic AAs [16]. AtAAP1/AtNAT2 (neutral amino acid transporter 2) facilitates AA import into the embryo [17, 18]. AtAAP8 contributes to the efficient uptake of AAs into the seed endosperm [19]. AtAAP3 and AtAAP5 may be involved in root AA absorption [11, 20]. AtAAP2 and AtAAP6 are reported to function in the xylem-phloem transfer of AAs [21, 22].

The allotetraploid Brassica napus $\left(\mathrm{A}_{n} \mathrm{~A}_{n} \mathrm{C}_{n} \mathrm{C}_{n}, 2 n=4 x=\right.$ $38)$ is the second most important oleaginous crops worldwide, and it originates from spontaneous hybridization of the diploid Brassica rapa $\left(\mathrm{A}_{\mathrm{r}} \mathrm{A}_{\mathrm{r}}, 2 n=2 x=20\right)$ and Brassica oleracea $\left(\mathrm{C}_{\mathrm{o}} \mathrm{C}_{\mathrm{o}}, 2 n=2 x=18\right)$ [23-25]. B. napus has a relatively higher demand for mineral nutrients, particularly $\mathrm{N}$, than grain crops to achieve optimal seed yield [26]. Indeed, despite its strong capacity of $\mathrm{N}$ import, $B$. napus has lower NUE than other major agriculture crops [27], which may be attributed to the fact that senescent leaves easily detach from rapeseed plants before $\mathrm{N}$ nutrients, specifically organic amide-N, have been fully remobilized to sink organs $[28,29]$. Therefore, improving the $\mathrm{N}$ remobilization efficiency in oilseed rape is important for NUE enhancement through molecular modulation of AA transporters, particularly AAPs.

However, few systematic analyses of $A A P S$ in $B$. napus have been available so far. In this study, we were aimed to (i) identify the genome-wide $A A P$ genes in $B$. napus, (ii) characterize the genomic characteristics and transcriptional responses of the $A A P$ gene members to $\mathrm{N}$ stresses, including nitrate limitation and ammonium toxicity, and (iii) investigate the transcriptional responses of $A A P s$ to other nutrient stresses, including phosphate limitation, boron deficiency, cadmium toxicity, and salt stress. The genome-wide identification and molecular characterization of the $A A P$ members indicated their evolutionary conservation and functional divergence between allotetraploid rapeseed and the model Arabidopsis. The global landscapes of the $A A P S$ might provide comprehensive insights into the AA import and translocation in allotetraploid rapeseed under diverse nutrient stresses.

\section{Results}

\section{Genome-wide identification of $A A P$ genes}

To identify the AAP family members in Brassica species, we used the AA sequences of AtAAPs to perform BLASTp queries against the genome databases of $B$. rapa ('Chiifu-401'), B. oleracea ('TO1000'), and B. napus ('Darmor-bzh'). The query results revealed great differences in the $A A P$ homolog number occurred during the evolutionary process of Brassica species (Table 1). As shown in Table 1, AAPs had eight members (AAP1$A A P 8)$ in the model $A$. thaliana, and each AAP member only had a single copy. A total of 19,17 , and $34 A A P$ homologs were identified in $B$. rapa, $B$. oleracea, and $B$. napus, respectively. It can be found that the number of $A A P s$ in $B$. napus was similar to the sum of $A A P S$ in both $B$. rapa and $B$. oleracea. Therefore, it could be concluded that most of the AAPs were retained during the alloploidy formation of $B$. napus. In $B$. napus, the number of $A A P s$ varied from one (BnaCn.AAP6) to nine (BnaAAP8s) with an average of more than four homologs for each member. The difference in the $A A P$ number might suggest their differential expansion patterns of $B n a A A P s$ during the allopolyploidy process of $B$. napus. 
Table 1 Copy number of the amino acid permease (AAP) genes in Arabidopsis and three Brassica species

\begin{tabular}{|c|c|c|c|c|}
\hline Item & Arabidopsis thaliana (125 Mb) & Brassica rapa (465 Mb) & Brassica oleracea (485 Mb) & Brassica napus (1130 Mb) \\
\hline$\overline{A A P 1}$ & 1 & 3 & 2 & 6 \\
\hline$A A P 2$ & 1 & 2 & 2 & 4 \\
\hline AAP3 & 1 & 1 & 1 & 3 \\
\hline AAP4 & 1 & 2 & 2 & 4 \\
\hline AAP5 & 1 & 3 & 3 & 5 \\
\hline AAPG & 1 & 1 & 1 & 1 \\
\hline$A A P 7$ & 1 & 1 & 1 & 2 \\
\hline$A A P B$ & 1 & 6 & 5 & 9 \\
\hline Total & 8 & 19 & 17 & 34 \\
\hline
\end{tabular}

\section{Genomic distribution and gene expansion of BnaAAPs}

Through physical position identification, we found that eight $A A P s$ in Arabidopsis showed strong preference for chromosomal distribution: four of them are located on the Chr. 01 (AtAAP1, AtAAP3, AtAAP5 and AtAAP8) and the other four on Chr. 05 (AtAAP2, AtAAP4, AtAAP6 and AtAAP7) (Table 2, Fig. 1a). To further explore the genomic distribution and gene expansion of $B n a A A P s$, we retrieved the DNA sequences of BnaAAPs in the Brassica and CNS-Genoscope databases. Different from the intensive distribution of AtAAPs, the BnaAAPs were physically scattered on 12 chromosomes (A subgenome: A1, A2, A3, A6, A7, A9, and A10; C subgenome: C1, C2, C3, C6, and C9) (Table 2). In the A subgenome of B. napus, Chromosome A9 exhibits the largest chromosome size and the second largest gene number according to the rapeseed genome annotation (http:// www.genoscope.cns.fr/brassicanapus/). Our study showed that Chromosome A9 has the largest BnaAAP gene number, i.e. four, including BnaA9.AAP1 (BnaA09g14700D), BnaA9.AAP7 (BnaA09g05130D), BnaA9.AAP8a (BnaA09g57230D), and BnaA9.AAP8b (BnaA09g57240D) (Table 2). In the $\mathrm{C}$ subgenome, more AAPs were identified on both Chromosome C6 and C8 than on the other seven chromosomes (C1-C5, C7, and C9). The homolog number of eight BnaAAP family genes varies from one to three, and they are relatively evenly distributed on different chromosomes (Table 2).

Gene family expands mainly via three pathways: tandem duplication, segmental duplication, and wholegenome duplication [30]. The B. napus progenitor diploids (B. rapa and $B$. oleracea) are ancient polyploids, and large-scale chromosomal rearrangements have occurred since their evolution from Amborella trichopoda, a low chromosome number progenitor [31]. Comparative genomics reveals that the Arabidopsis genome is divided into 24 ancestral crucifer blocks, which are labeled A-X [32]. Table 2 shows that the AtAAP genes and their corresponding homologs in $B$. napus are located in the same chromosomal blocks. To further understand the expansion patterns of BnaAAPs, we investigated their duplication events. The results showed that the $A A P$ family members in $B$. napus were derived from the corresponding ancestors in $B$. rapa or $B$. oleracea, except $B n a A A P 8 s$, which expanded potentially through tandem duplication (Figs. 1b and 2). Based on the above result, we presumed that segmental duplication might be a main contributor to the BnaAAP family expansion.

\section{Phylogeny analysis of BnaAAPs}

To elucidate the molecular evolution and phylogenetic relationships among the AAP proteins, we constructed two unrooted phylogenetic trees involving AtAAPs and their homologs in Brassica species (Fig. 2). In Arabidopsis, according to the phylogeny relationships, we made the first attempt to classify the AAP family members into three major clades: Clade I (AtAAP1, AtAAP6, and AtAAP8), Clade II (AtAAP2, AtAAP3, AtAAP4, and AtAAP5), and Clade III (AtAAP7) (Fig. 2a). Further, we performed a phylogeny analysis of 78 AAP proteins in $A$. thaliana and three Brassica crop species, including $B$. rapa, $B$. oleracea, and $B$. napus. The phylogenetic tree was also subclassified into eight smallest categories, and the AAP members from different species were closely clustered with their corresponding homologs in $A$. thaliana (Fig. 2b). What is noteworthy, the $A A P$ genes of $B$. napus were clustered into the smallest clades with their corresponding homologs in the diploid ancestor $B$. rapa or B. oleracea (Additional file: Figure S1). The result indicated that the AAP proteins divergence was prior to the Brassica speciation. We also observed that most of the AAP proteins within each subfamily had very short branch lengths (Fig. 2b), suggesting a recent genetic divergence.

\section{Molecular characterization of BnaAAPs}

To understand the molecular characteristics of the BnaAAP proteins, we calculated the physicochemical parameters of each BnaAAP protein using ExPASy. The results showed that most proteins in the same AAP 
Table 2 Molecular characterization of the amino acid permease (AAP) genes in Arabidopsis thaliana and Brassica napus

\begin{tabular}{|c|c|c|c|c|c|c|c|c|c|}
\hline Gene ID & Gene name & Block & CDS (bp) & $\begin{array}{l}\text { Exon/ } \\
\text { intron }\end{array}$ & $\begin{array}{l}\text { Amino acid } \\
\text { (aa) }\end{array}$ & Ka & Ks & $\mathrm{Ka} / \mathrm{Ks}$ & $\begin{array}{l}\text { Divergent time } \\
\text { (Mya) }\end{array}$ \\
\hline At1g58360 & AtAAP1 & $\mathrm{D}$ & 1458 & $6 / 5$ & 485 & & & & \\
\hline BnaA01g21750D & BnaA1.AAP1 & $\mathrm{D}$ & 1524 & $6 / 5$ & 507 & 0.0394 & 0.3931 & 0.1002 & 13.10 \\
\hline BnaA03g59400D & BnaA3.AAP1 & $\mathrm{D}$ & 1623 & $8 / 7$ & 540 & 0.0565 & 0.3987 & 0.1417 & 13.29 \\
\hline BnaA09g14700D & BnaA9.AAP1 & $\mathrm{D}$ & 1440 & $6 / 5$ & 479 & 0.0466 & 0.4043 & 0.1153 & 13.48 \\
\hline BnaC01g42990D & BnaC1.AAP1 & $\mathrm{D}$ & 1524 & $6 / 5$ & 507 & 0.0405 & 0.3747 & 0.1081 & 12.49 \\
\hline BnaC04g18440D & BnaC4.AAP1 & $\mathrm{D}$ & 1455 & $6 / 5$ & 484 & 0.0489 & 0.3854 & 0.1269 & 12.85 \\
\hline BnaCnng25620D & BnaCn.AAP1 & $\mathrm{D}$ & 1440 & $6 / 5$ & 479 & 0.0489 & 0.3965 & 0.1233 & 13.22 \\
\hline At5g09220 & AtAAP2 & $\mathrm{R}$ & 1482 & $6 / 5$ & 493 & & & & \\
\hline BnaA03g02650D & BnaA3.AAP2 & $\mathrm{R}$ & 1464 & $7 / 6$ & 487 & 0.0293 & 0.4707 & 0.0622 & 15.69 \\
\hline BnaA10g22670D & BnaA10.AAP2 & $\mathrm{R}$ & 1458 & $6 / 5$ & 485 & 0.0366 & 0.5027 & 0.0728 & 16.76 \\
\hline BnaC03g03750D & BnaC3.AAP2 & $\mathrm{R}$ & 1464 & $6 / 5$ & 487 & 0.0313 & 0.4676 & 0.0669 & 15.59 \\
\hline BnaC09g47230D & BnaC9.AAP2 & $\mathrm{R}$ & 1458 & $6 / 5$ & 485 & 0.0437 & 0.4787 & 0.0913 & 15.96 \\
\hline At1g77380 & AtAAP3 & $E$ & 1431 & $7 / 6$ & 476 & & & & \\
\hline BnaA07g33510D & BnaA7.AAP3 & $E$ & 1431 & $7 / 6$ & 476 & 0.0374 & 0.3945 & 0.0948 & 13.15 \\
\hline BnaC06g38080D & BnaC6.AAP3a & $E$ & 1431 & $7 / 6$ & 476 & 0.0386 & 0.3865 & 0.0999 & 12.88 \\
\hline BnaC06g38090D & BnaC6.AAP3b & $E$ & 1431 & $7 / 6$ & 476 & 0.0408 & 0.3847 & 0.1061 & 12.82 \\
\hline At5g63850 & AtAAP4 & $x$ & 1401 & $6 / 5$ & 466 & & & & \\
\hline BnaA02g33930D & BnaA2.AAP4 & $x$ & 1401 & $6 / 5$ & 466 & 0.0304 & 0.5479 & 0.0555 & 18.26 \\
\hline BnaA06g22970D & BnaA6.AAP4 & $x$ & 1401 & $5 / 4$ & 466 & 0.0392 & 0.4567 & 0.0858 & 15.22 \\
\hline BnaC02g42740D & BnaC2.AAP4 & $x$ & 1401 & $5 / 4$ & 466 & 0.0306 & 0.5398 & 0.0567 & 17.99 \\
\hline BnaC03g50500D & BnaC3.AAP4 & $x$ & 1401 & $5 / 4$ & 466 & 0.0423 & 0.4367 & 0.0969 & 14.56 \\
\hline At1g44100 & AtAAP5 & $C$ & 1443 & $5 / 4$ & 480 & & & & \\
\hline BnaAnng17090D & BnaAn.AAP5 & $C$ & 1248 & $4 / 3$ & 415 & 0.0804 & 0.4964 & 0.1620 & 16.55 \\
\hline BnaA08g04440D & BnaA8.AAP5 & $C$ & 1446 & $5 / 4$ & 481 & 0.0840 & 0.5449 & 0.1542 & 18.16 \\
\hline BnaA05g18660D & BnaA5.AAP5 & C & 1464 & $5 / 4$ & 487 & 0.0801 & 0.5204 & 0.1539 & 17.35 \\
\hline BnaA10g08840D & BnaA10.AAP5 & $C$ & 1431 & $5 / 4$ & 476 & 0.1206 & 0.5015 & 0.2405 & 16.72 \\
\hline BnaC06g00580D & BnaC6.AAP5 & C & 1431 & $5 / 4$ & 476 & 0.1149 & 0.4909 & 0.2341 & 16.36 \\
\hline At5g49630 & AtAAP6 & W & 1446 & $6 / 5$ & 481 & & & & \\
\hline BnaCnng14480D & BnaCn.AAP6 & W & 1440 & $6 / 5$ & 479 & 0.0560 & 0.4756 & 0.1177 & 15.85 \\
\hline At5g23810 & AtAAP7 & Q & 1404 & $6 / 5$ & 467 & & & & \\
\hline BnaA09g05130D & BnaA9.AAP7 & Q & 1413 & $7 / 6$ & 470 & 0.1092 & 0.3543 & 0.3082 & 11.81 \\
\hline BnaC09g04700D & BnaC9.AAP7 & Q & 1059 & $6 / 5$ & 352 & 0.1169 & 0.3813 & 0.3066 & 12.71 \\
\hline At1g10010 & AtAAP8 & A & 1428 & $6 / 5$ & 475 & & & & \\
\hline BnaA06g38000D & BnaA6.AAP8a & A & 1401 & $6 / 5$ & 466 & 0.1387 & 0.4977 & 0.2787 & 16.59 \\
\hline BnaA06g38010D & BnaA6.AAP8b & A & 1389 & $6 / 5$ & 462 & 0.1385 & 0.5880 & 0.2355 & 19.60 \\
\hline BnaA09g57230D & BnaA9.AAP8a & A & 1446 & $6 / 5$ & 481 & 0.1387 & 0.4977 & 0.2787 & 16.59 \\
\hline BnaA09g57240D & BnaA9.AAP8b & A & 1584 & $6 / 5$ & 527 & 0.1385 & 0.5880 & 0.2355 & 19.60 \\
\hline BnaC05g49200D & BnaC5.AAP8a & A & 1410 & $7 / 6$ & 469 & 0.1361 & 0.5426 & 0.2508 & 18.09 \\
\hline BnaC05g49210D & BnaC5.AAP8b & A & 1449 & $6 / 5$ & 482 & 0.1385 & 0.5880 & 0.2355 & 19.60 \\
\hline BnaC08g42410D & BnaC8.AAP8a & A & 1446 & $6 / 5$ & 481 & 0.1508 & 0.2216 & 0.6805 & 7.39 \\
\hline BnaC08g42420D & BnaC8.AAP8b & A & 1515 & $7 / 6$ & 504 & 0.1463 & 0.5326 & 0.2747 & 17.75 \\
\hline BnaC08g42430D & BnaC $8 . A A P 8 C$ & A & 1446 & $6 / 5$ & 481 & 0.1321 & 0.5257 & 0.2513 & 17.52 \\
\hline
\end{tabular}


(A)
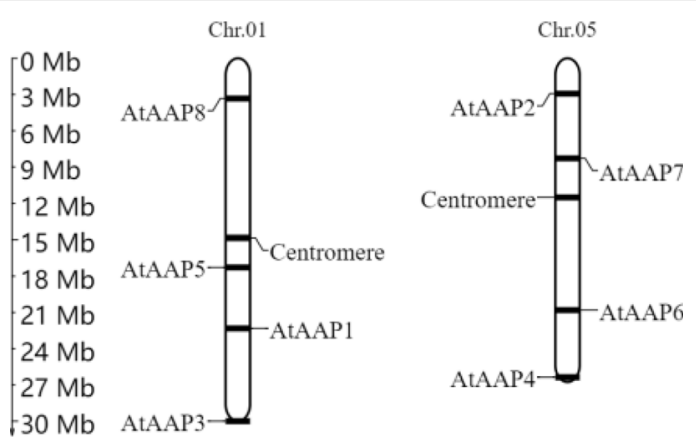

(B)
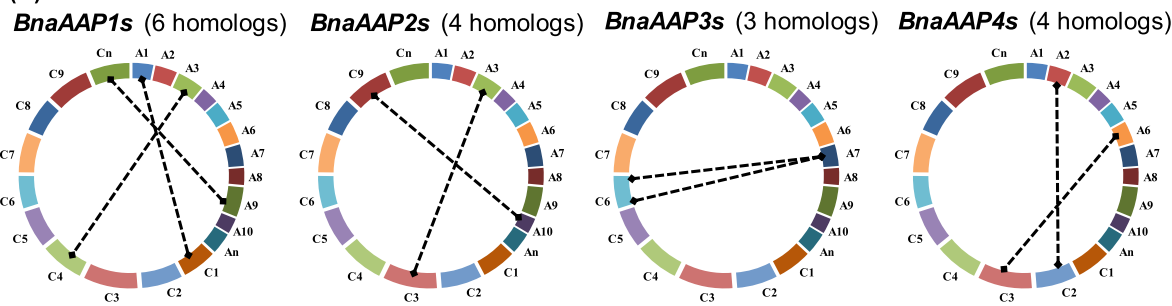

BnaAAP5s (5 homologs) BnaAAP6s (1 homolog)

BnaAAP7s (2 homologs) BnaAAP8s (9 homologs)
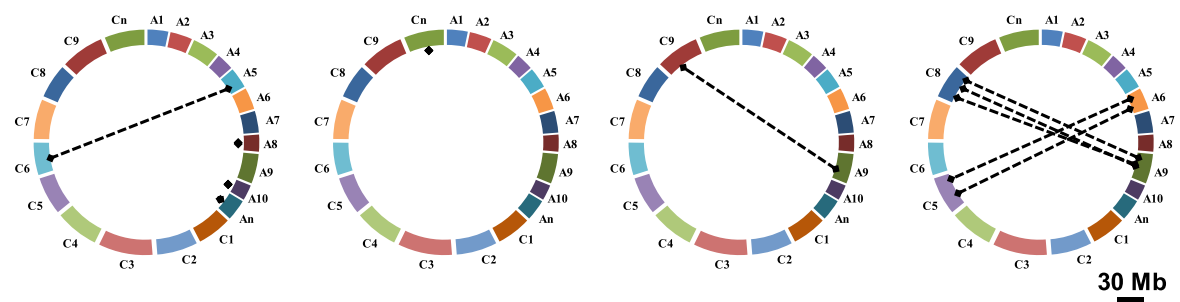

Fig. 1 Physical mapping and syntenic analysis of the amino acid permease (AAP) family genes in Arabidopsis thaliana and Brassica napus. a Genomic position of the Arabidopsis AAP genes (a) and their homologs in B. napus (b). The BnaAAP homologs between the An and Cn sub-genomes of rapeseed are connected by crashed lines. The length scale of B. napus chromosomes (An subgenome: A1-A10; Cn subgenome: C1-C9) is 30.0 Mb

(A)

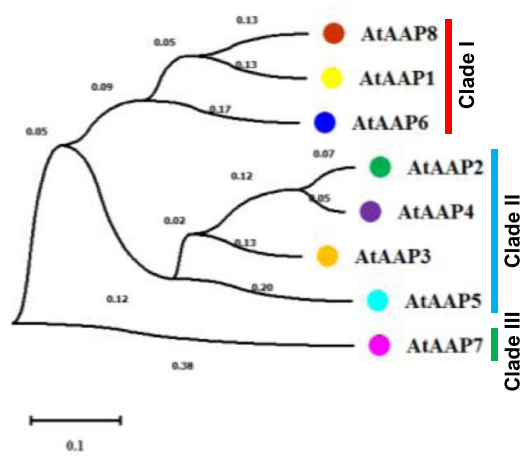

(B)

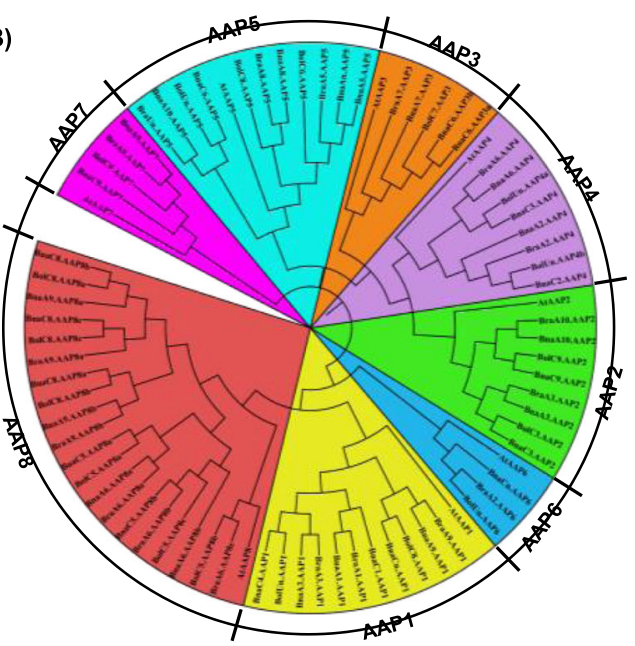

Fig. 2 Phylogeny analysis of the amino acid permease (AAP) genes in Arabidopsis thaliana and Brassica crops. a-b Phylogeny analysis of AtAAPS (a) and the AAPs in Brassica species (b), including A. thaliana, B. rapa, B. oleracea, and B. napus. The AAP protein sequences were multi-aligned using the ClustalW program, and then an unrooted phylogenetic tree was constructed using MEGA 6.06 with the neighbor-joining method. The percentages of replicate trees, in which the associated taxa clustered together in the bootstrap test (1000 replicates), are shown next to the branches. The tree is drawn to scale, with branch lengths in the same units as those of the evolutionary distances used to infer the phylogenetic tree. The evolutionary distances were computed with the Poisson correction method, and are in the units of the number of amino acid substitutions per site 
subfamily had similar parameters (Table 2). In total, the coding sequence (CDS) lengths of BnaAAPs varied from 1059 bp (BnaC9.AAP7) to 1584 bp (BnaA9.AAP8b), corresponding to the variation of the deduced AA number from 352 to 527 (Table 2). Most of the computed molecular weights (MWs) of the BnaAAP proteins ranged from $50.0 \mathrm{kD}$ to $60.0 \mathrm{kD}$, except that those of BnaA10.AAP5 (45.8 kD) and BnaC9.AAP7 (38.4 kD) were smaller than $50.0 \mathrm{kD}$ (Fig. 3a, Additional file 1: Table S1). The theoretical isoelectric points (pIs) of BnaAAPs varied from 6.19 (BnaA3.AAP1) to 9.28 (BnaA6.AAP8b), with most $>7.0$ except that of BnaA3.AAP1 (6.19) (Fig. 3b, Additional file 1: Table S1). The grand average of hydropathy (GRAVY) value is calculated as the sum of hydropathy values of the AAs divided by the protein length. The results showed that the GRAVY values of the BnaAAP members ranged from 0.360 (BnaC8.AAP8a) to 0.566 (BnaC5.AAP8b) (Fig. 3c, Additional file 1: Table S1). Therefore, all the BnaAPPs were presumed to be hydrophobic. The instability indices (IIs) values of all the BnaAAPs were $<40.0$ (Fig. 3d, Additional file 1: Table S1), which showed strong protein stability. The online WoLF PSORT was used to predict the subcellular localization of eight AtAAPs and 34 BnaAAPs. The result indicated that they were localized in the plasma membrane, suggesting that they might be responsible for the trans-membrane transport of AAs. We used the TMHMM tool to characterize the transmembrane structures of AAPs in A. thaliana and $B$. napus, and found that AtAAPs and BnaAAPs had nine to ten membrane-spanning regions (Additional file 1: Figure S2). In detail, the AtAAP5/BnaAAP5s, AtAAP6/ BnaAAP6s, and AtAAP7/BnaAtAAP7s had ten transmembrane regions, and the other five subgroup members had nine membrane-spanning regions Additional file 1: Figure S2). Similarly to the AtAAPs without signal peptides (Additional file 1: Figure S3), the BnaAPPs were observed to have no signal peptides, either. The Recombinant Protein Solubility Prediction (version 2009) indiated that the recombinant BnaAAPs would be insoluble when these proteins were overexpressed in E. coli.

\section{Identification of evolutionary selection pressure on BnaAAPs}

To characterize selection pressure on the BnaAAPs during the evolutionary process, we used the orthologous $A A P$ gene pairs between $B$. napus and A. thaliana to determine the values of synonymous (Ks) and nonsynonymous (Ka) nucleotide substitution rates, and $\mathrm{Ka} /$ Ks (Table 2). The Ka values of BnaAAPs ranged from 0.0293 (BnaA3.AAP2) to 0.1508 (BnaC8.AAP8a) with an average of 0.05 , and the Ks values of BnaAAPs ranged

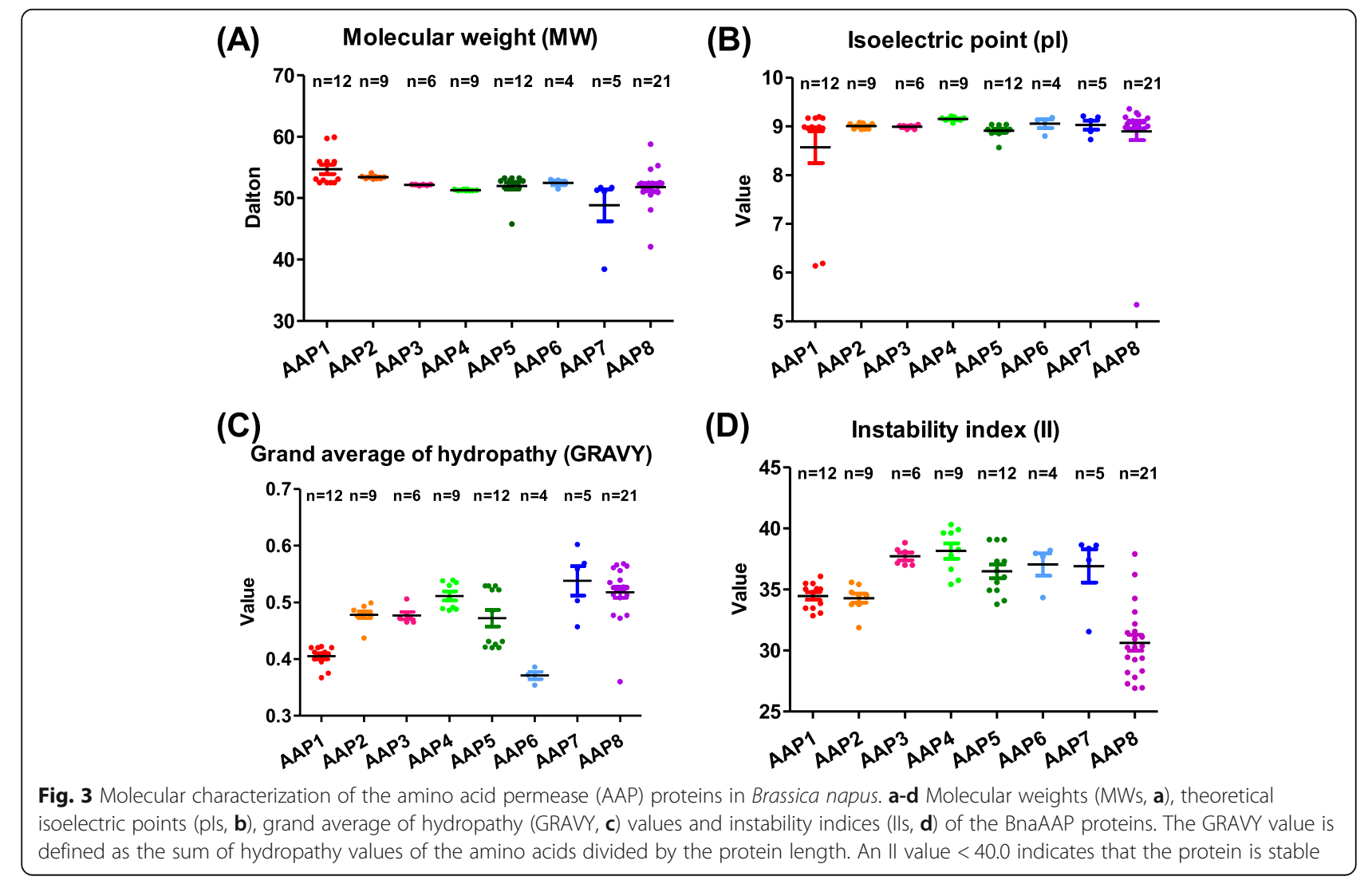


from 0.2216 (BnaA3.AAP2) to 0.5880 (BnaC8.AAP8a) with an average of 0.5199. Further, we found that all the $\mathrm{Ka} / \mathrm{Ks}$ values of BnaAAPs were $<1.0$ (Table 2). Therefore, we presumed that the BnaAAPs might have experienced a very strong negative selection to preserve their function.

The Ks values of the duplicated homologs among gene families are usually presumed to be molecular clocks, and these Ks values are assumed to be constant over time. It has been reported that the segregation between the model Arabidopsis and its-derived Brassica plants occurred 1220 million years ago (Mya) [33]. In this study, our results showed that most of BnaAAPs might diverge from AtAAPs approximately $12.0-18.0$ Mya except BnaC8.AAP8a, implying that the Brassica speciation was accompanied by the divergence of the $A A P$ genes.

\section{Conserved domain, exon-intron organization, gene} interaction, and transcriptional regulatory analysis of BnaAAPs

AA residues are thought to be functionally or structurally significant if they are evolutionarily conserved. The output of MEME showed that the eight BnaAAP subfamilies had conserved domains (Fig. 4a). Further, we identified that these conserved domains belong to the amino acid transporter family, which is part of the solute carrier (SCL) superfamily proteins with solute binding domains (Fig. 4a). Among the ten conserved domains we defined, the AA sequences of Motif I and IX exhibited the highest identity (Fig. 4b), thereby which could be used as the identification indicators of the AAP family members.

To further identify the protein(s) potentially interacting with the AAP family members, we constructed a protein interaction network involving direct (physical) and indirect (function) association by using the STRING database based on either known experimental or predicted interactions. As shown in Fig. 5, all the AAP proteins consistently interacted with CAT6, a cation AA transporter (CAT) belonging to the AA polyamine choline (APC) family [34]. Besides, some other AA transporters, such as CAT8, SIAR1 (Silique Are Red 1), and the UMAMIT (Usually Multiple Acids Move In and out Transporters) family members, were also observed to interact with the AAP proteins (Fig. 5).

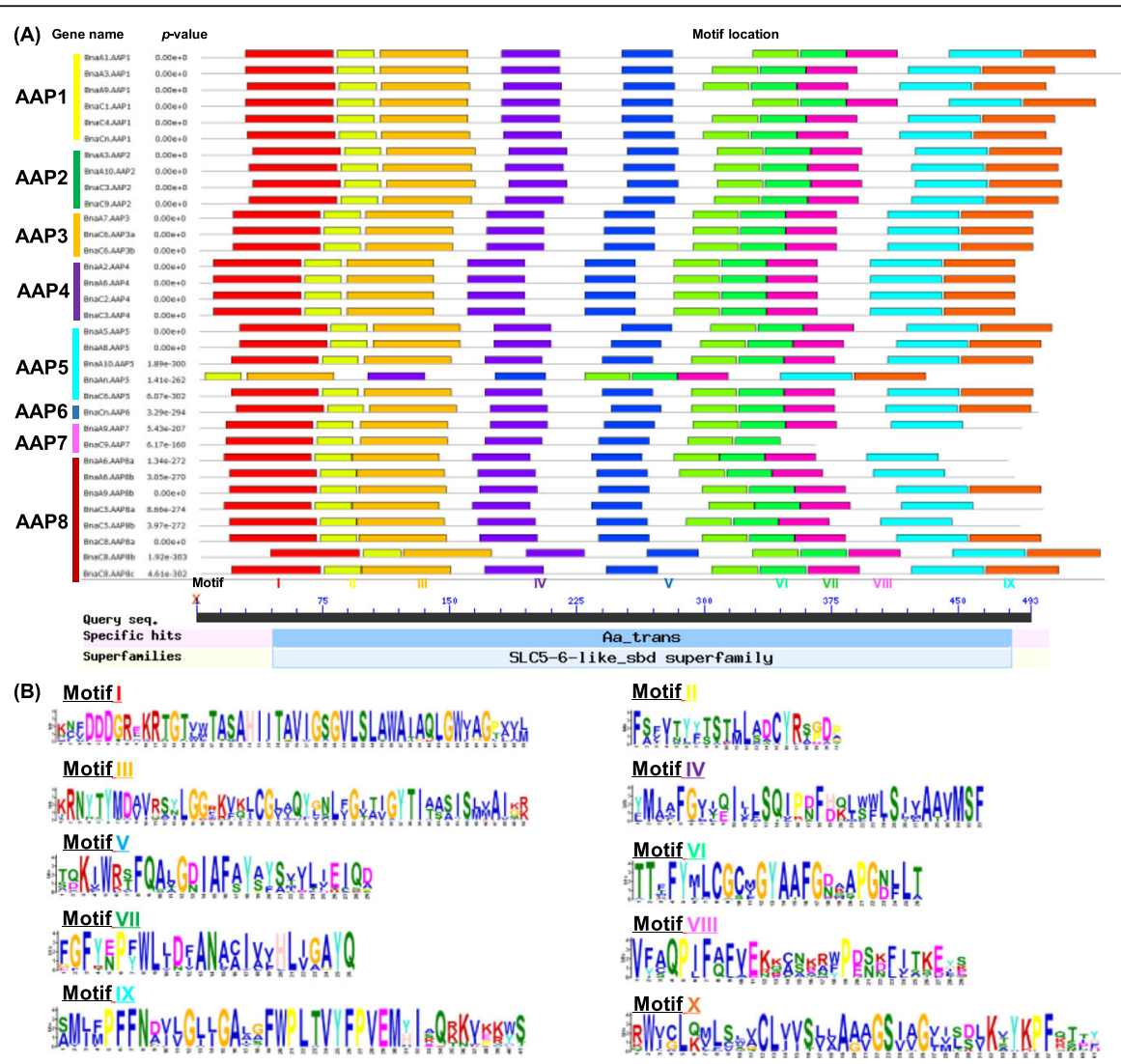

Fig. 4 Identification and characterization of the conserved motifs in the amino acid permease (AAP) proteins in Arabidopsis thaliana and Brassica napus. Molecular identification (a) and sequence characterization (b) of the conserved motifs in the AAP proteins in A. thaliana and B. napus. In $\mathbf{a}$, the boxes with different colors indicate different conserved motifs (motif 1-15), and grey lines represent the AAP protein regions without detected motifs. Aa_trans indicates the amino acid transport regions; SCL5-6-like_sbd refers to the solute carrier (SCL) families 5 and 6-like proteins with solute binding domain (sbd). In $\mathbf{b}$, the larger the fonts, the more conserved the motifs 


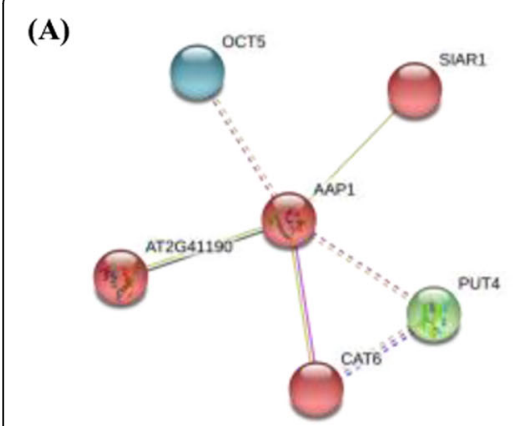

(D)

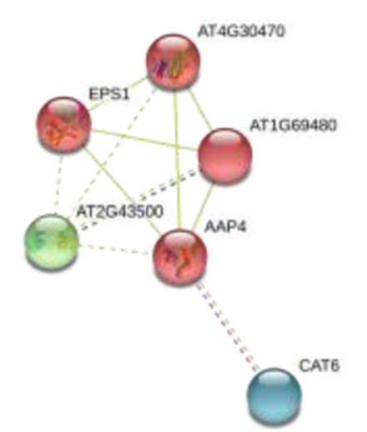

(B)

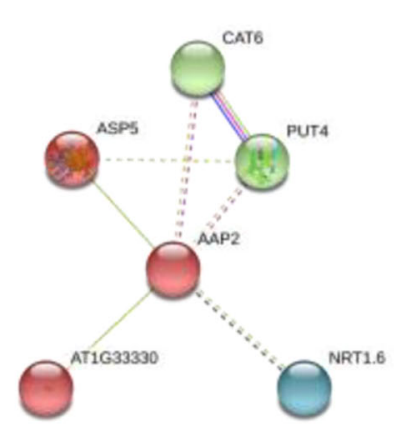

(E)

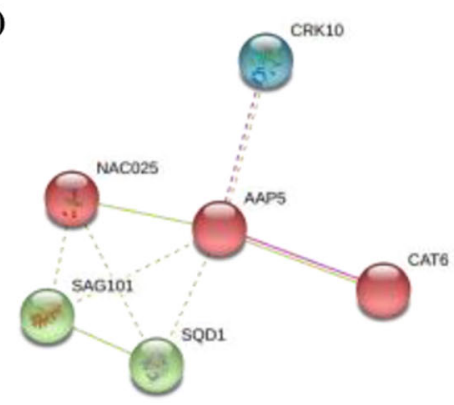

(C)

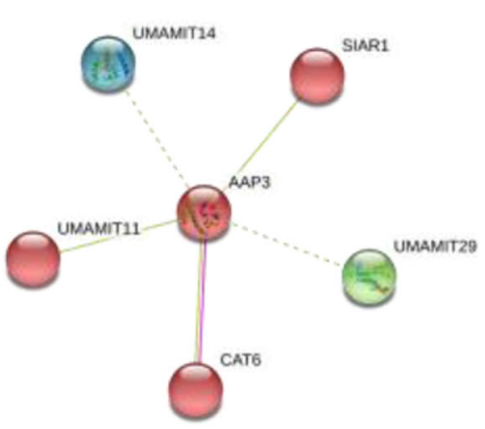

(F)

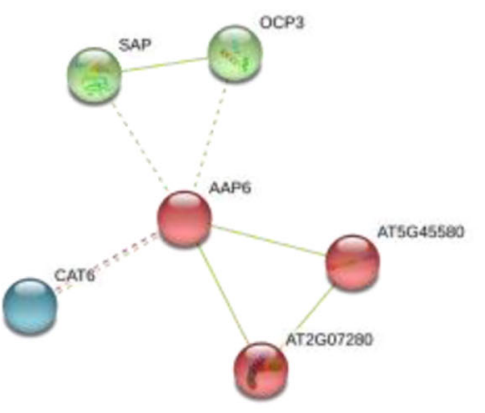

(G)
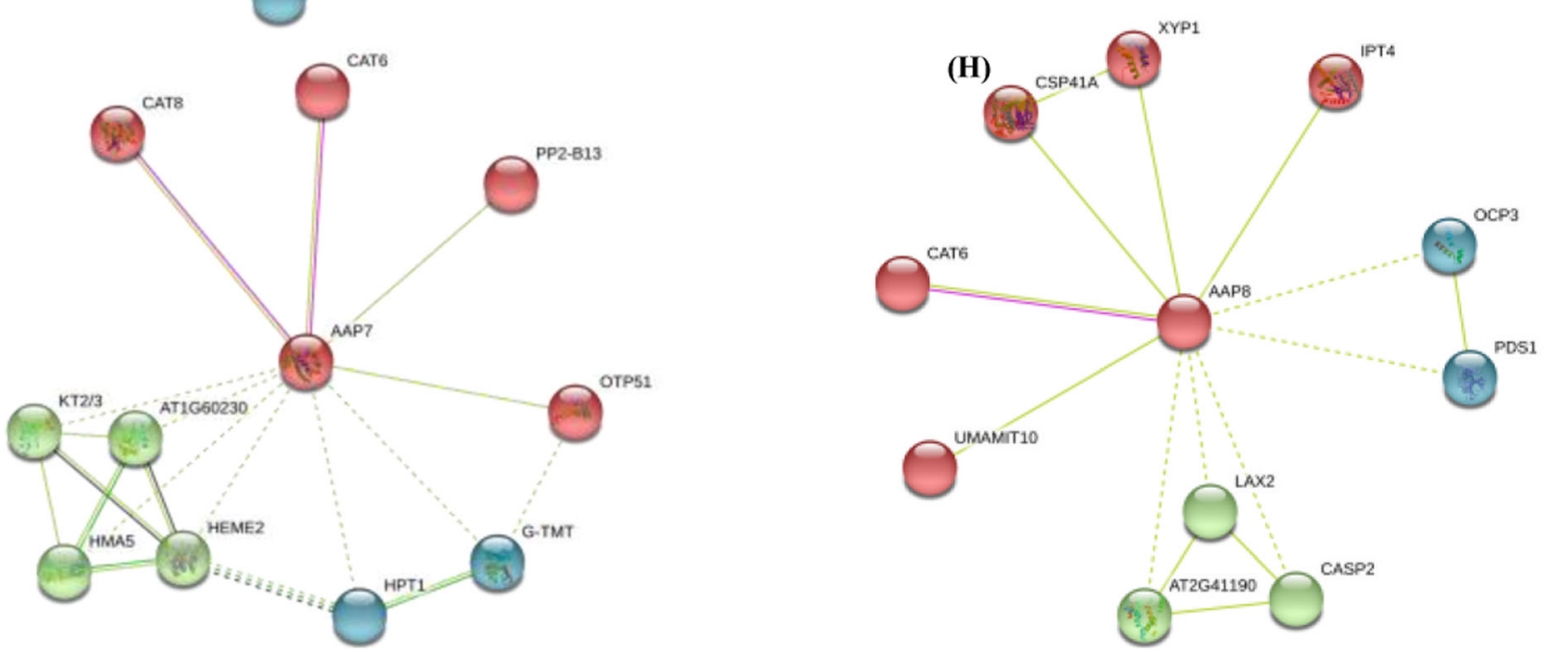

Fig. 5 Protein-protein interaction networks of the amino acid permease (AAP) proteins in Brassica napus. The interaction networks of the AAP1 $(\mathbf{a})$, AAP2 (b), AAP3 (c) AAP4 (d), AAP5 (e), AAP6 (f), AAP7 (g) and AAP8 (h) and other proteins were constructed by the STRING web-server. Network nodes represent proteins. The network is clustered into three clusters, which are represented with red, green, and blue nodes, respectively. Colored nodes: query proteins and first shell of protein interactors; white nodes: the second shell of protein interactors. Empty nodes: proteins of unknown 3D structure; filled nodes: some 3D structure is known or predicted. Edges represent protein-protein associations

The exon-intron number and organization are indicative of evolutionary imprints within gene families. Considering this, we identified the gene structures of BnaAAPs by comparing the genomic DNA sequences with their corresponding CDSs. As shown in Fig. 6(a, b) and Table 2, in general, most AtAAPs had similar gene structures to their homologs in B. napus, which indicated their conserved functionality between the ancestor Arabidopsis and $B$. napus. However, we also observed some structure variations within a BnaAAP subgroup (Table 2). The exon/intron number variations, potentially caused by alternative splicing, might contribute to the functional differentiation of different BnaAAP members.

Transcription factors (TFs), binding to cis-acting regulatory elements (CREs) in the promoters of their target genes, play important roles in the transcriptional regulation [36]. To identify the core TFs regulating BnaAAPs, the 2.0-kb upstream sequences of the BnaAAP start condons were used to explore the over-accumulated CREs (Fig. 6). The results showed that all the BnaAAP family genes had various types of CREs in their promoter 

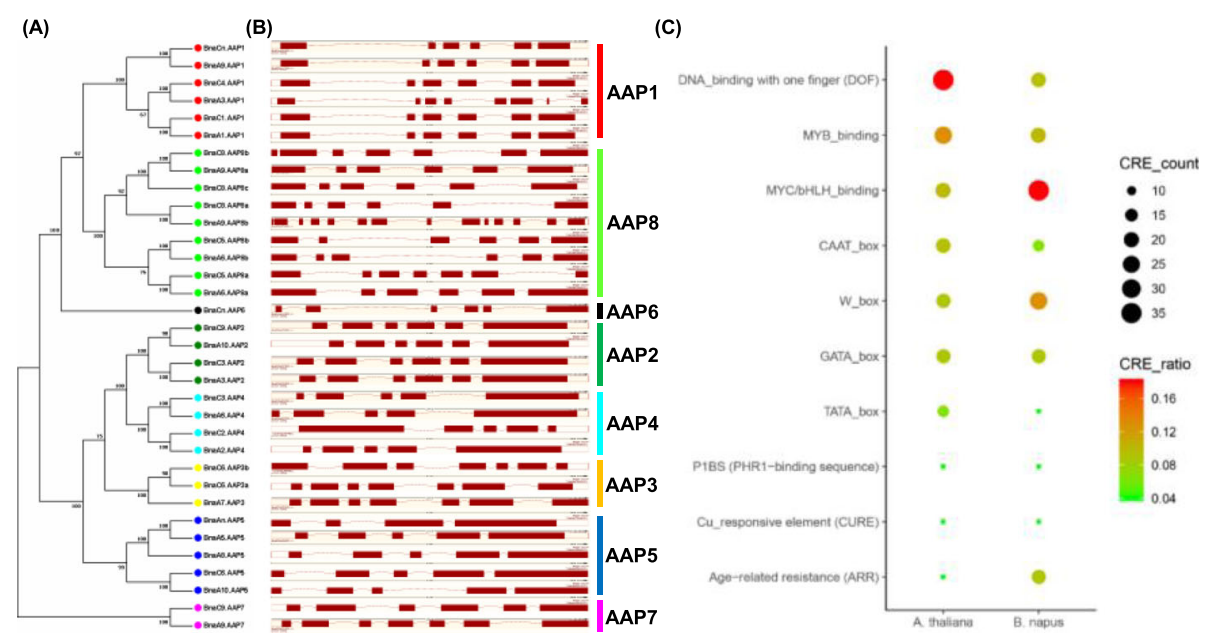

Fig. 6 Phylogeny relationship (a), exon-intron organization $\mathbf{b}$ ) of the amino acid permease (AAP) genes and enrichment analysis (c) of the cisacting regulatory elements (CREs) in the AAP promoter regions in Brassica napus. For each AAP, a 2.0-kb genomic sequence in the upstream from the start codon (ATG) was downloaded from the TAIR (https://www.arabidopsis.org/) website and B. napus Genome Browser (http://www. genoscope.cns.fr/brassicanapus/) [24]. Subsequently, we submitted these sequences to the PLACE v. 30.0 (http://www.dna.affrc.go.jp/PLACE/) program [35] to identify putative CREs and calculate the CRE number along the $2.0 \mathrm{~kb}$ gene promoter region. In the scatter plot of $\mathbf{c}$, the larger the circle sizes, the more the corresponding CREs

regions, which suggested that the complicated regulatory networks of BnaAAPs might be involved in their transcriptional regulation. Apart from the common CREs, such as the TATA box and the CAAT box, we also identified that three terms, namely, GATA-box, W-box (TGAC), and MYC/bHLH-binding elements were most highly enriched in the BnaAAP promoter regions (Fig. 6c). In both Arabidopsis and B. napus, the DNA-binding with one finger (Dof), MYB-binding, and MYC/bHLHbinding elements were highly enriched (Fig. 6c). These results showed that on one hand, there Arabidopsis and $B$. napus shared the common regulatory mechanisms of $A A P s$, on the other hand, these two species had their own specific regulatory mechanisms.

\section{Transcriptional analysis of BnaAAPs under diverse nutrient stresses}

To identify the roles of BnaAAPs in regulating rapeseed against various nutrient stresses, we examined their transcriptional responses under these circumstances. First, we investigated the transcriptional patterns of AtAAPs in various tissues through the TAIR eFP Browser. The results showed that the AtAAP1, AtAAP2, AtAAP4, AtAAP5, and AtAAP6 genes were highly expressed in cauline and senescent leaves (Additional file 1: Figure S4A, B, D, E, and F), which indicated that these genes might play pivotal roles in the translocation of AAs from source leaves to sink organs. Furthermore, both AtAAP3 and AtAAP8 had the highest expression levels in the embryo seeds (Additional file 1: Figure $\mathrm{S} 4 \mathrm{C}$ and $\mathrm{H}$ ), suggesting that they might be involved in seed development. The preferential expression of AtAAP7 in the second internodes (Additional file 1: Figure S3G) suggested its participation in long-distance translocation of amide- $\mathrm{N}$ nutrients. The differential expression of AAPs implied their specific roles in plant growth and development.

The above-mentioned results indicated that multiple copies of each $A A P$ homolog occurred in allotetraploid rapeseed (Fig. 1), and that transcriptional identification of the core $A A P$ members were very important for the in-depth understanding of the BnaAAP function. Rapeseed has a high demand for $\mathrm{N}$ nutrients whereas it shows very low NUE [26, 27]. Under $\mathrm{N}$ stresses, the metabolic profiles of AAs were significantly altered in plants [37]. Under nitrate limitation condition, 26 members of 34 BnaAAPs were differentially expressed in rapeseed plants compared with the condition of sufficient nitrate supply (Fig. 7, Additional file 1: Tables S2, S3). In detail, most of the differentially expressed genes (DEGs) were upregulated in the shoots or roots under nitrate deficiency except that BnaA7.AAP3 and BnaCn.AAP6 were downregulated in the roots. It should be noted that among the eight BnaAAP subfamilies, the expression of all the BnaAAP1s was consistently induced in both the shoots and roots (Fig. 7a). Under ammonium toxicity, we identified a total of 26 BnaAAP DEGs in the shoots and roots relative to the condition of nitrate sufficiency (Fig. 8). In the shoots, we found that the DEGs of only BnaAAP1s and BnaAAP8s showed higher expression levels under nitrate sufficiency than under ammonium toxicity (Fig. 8a, h), and the DEGs of other $B n a A A P s$ were upregulated only when ammonium was supplied as the sole $\mathrm{N}$ nutrient source (Fig. 8b-g). In the roots, the expression of most family members 

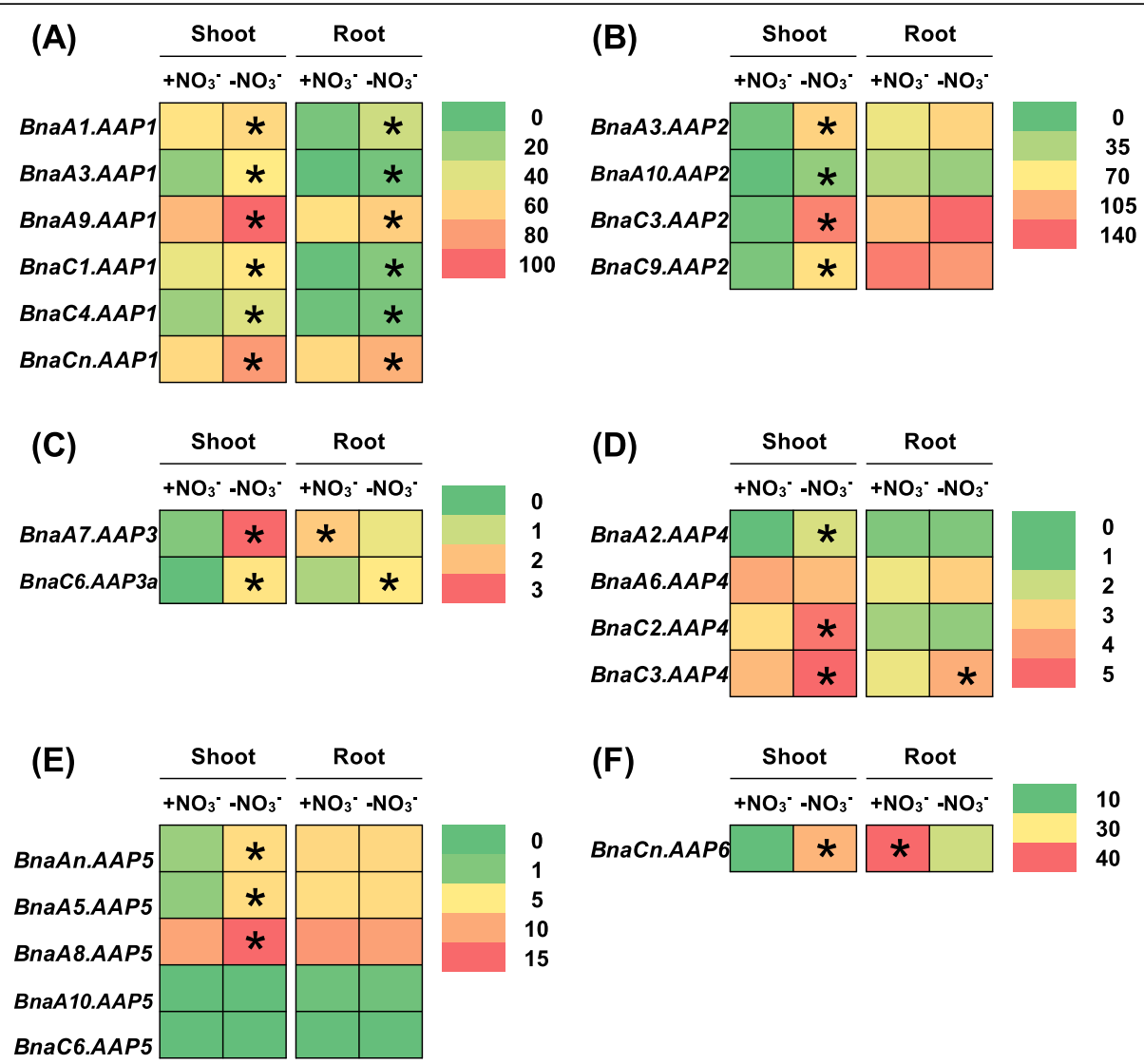

(F)
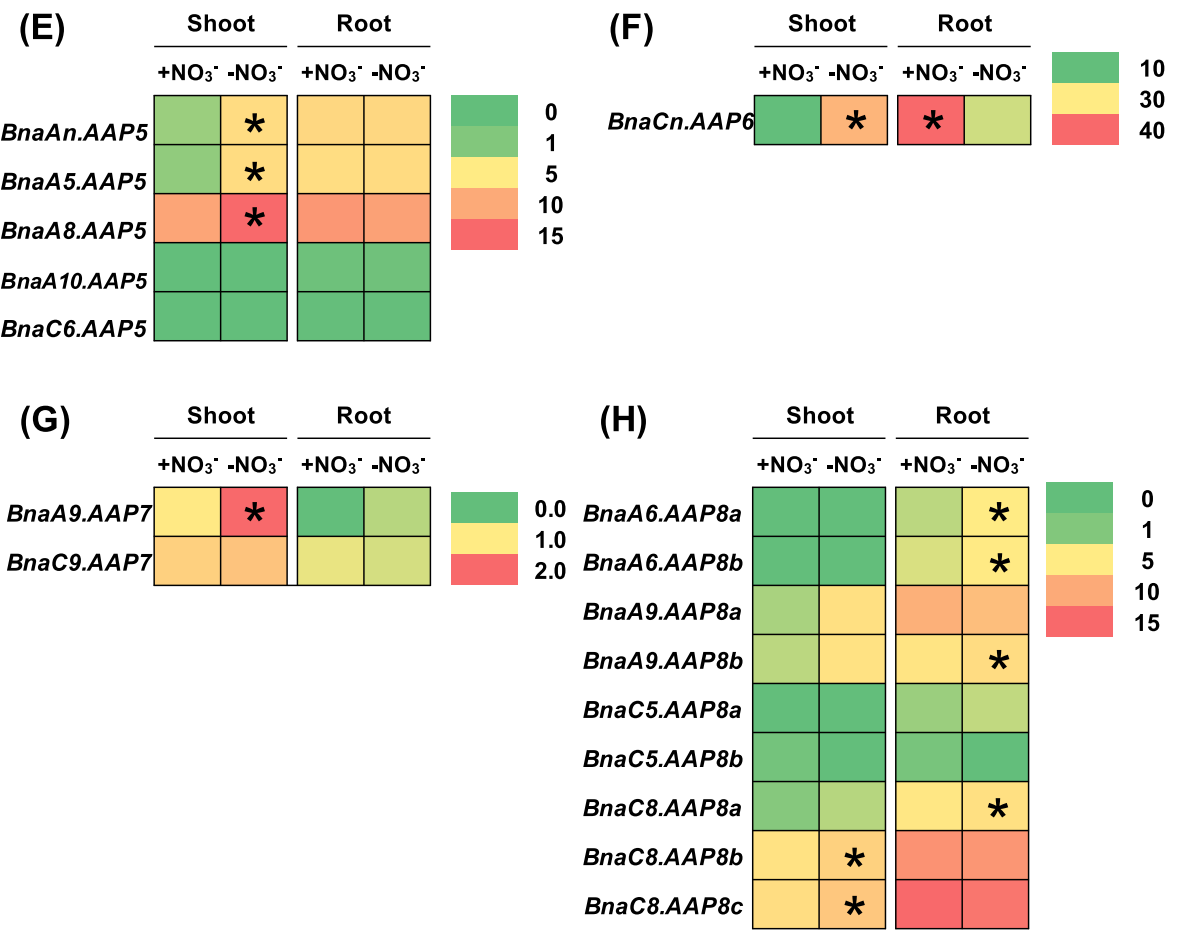

Fig. 7 The qRT-PCR-assisted transcriptional characterization of the amino acid permease (AAP) genes in Brassica napus under different nitrate $\left(\mathrm{NO}_{3}{ }^{-}\right)$supply levels. Differential expression of the BnaAAP1 (a), BnaAAP2(b), BnaAAP3 (c), BnaAAP4 (d), BnaAAP5(e), BnaAAP6 (f), BnaAAP7 (g) and BnaAAP8 (h) genes under high and low nitrate conditions. For the transcriptional analysis, the 7-d-old uniform $B$. napus seedlings after seed germination were cultivated under high $(+, 6.0 \mathrm{mM})$ nitrate for $10 \mathrm{~d}$, and then were transferred to low $(-, 0.30 \mathrm{mM})$ nitrate for $3 \mathrm{~d}$ until sampling. The shoots and roots were individually sampled, and each sample includes three independent biological replicates. The significance level of $P<$ 0.05 is used as the threshold to identify the differential expression of BnaAAPs under high and low nitrate treatments. The differentially expressed genes with higher expression between different treatments in the shoots or roots are indicated with asterisks

(BnaAAP2s, BnaAAP4s, BnaAAP5s, BnaAAP6s, and $B n a A A P 8 s$ ) was induced under ammonium toxicity (Fig. $8 \mathrm{~b}, \mathrm{~d}, \mathrm{e}, \mathrm{f}, \mathrm{h}$ ) whereas the differential expression profiling of BnaAAP1s (except BnaA3.AAP1) and BnaAAP3s presented the opposite pattern (Fig. 8a, c).
Phosphorus is one of the mineral nutrients required for plant growth, and it is widely used as fertilizers in agricultural production. $\mathrm{Hu}$ et al. [38] found that phosphate availability modulates the expression of nitrateresponsive genes, and that the NRT1.1B-SPX4 module is 


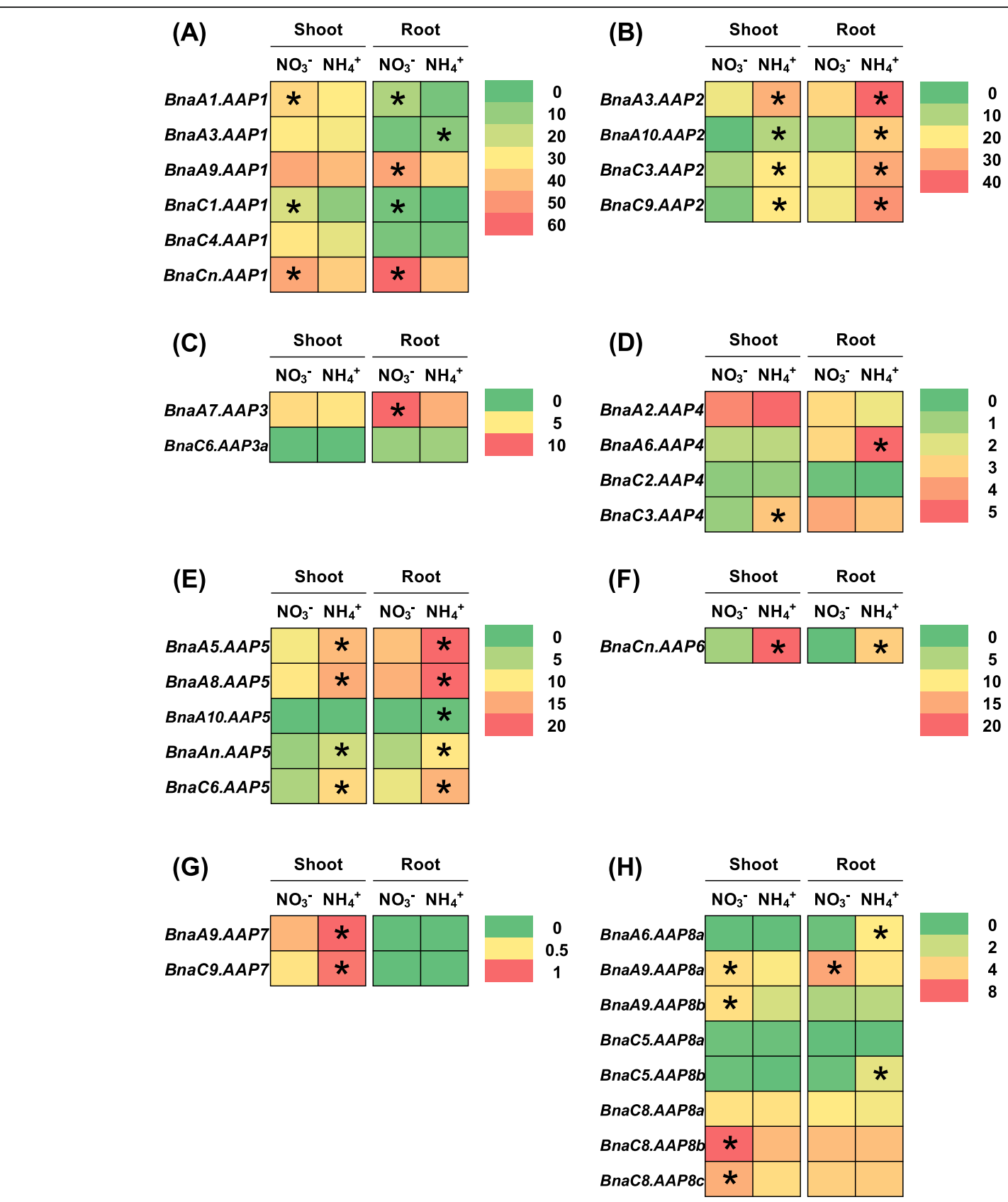

Fig. 8 The qRT-PCR-assisted transcriptional characterization of the amino acid permease (AAP) genes in Brassica napus under different nitrogen (N) form conditions. Differential expression of the BnaAAP1 (a), BnaAAP2 (b), BnaAAP3 (c), BnaAAP4 (d), BnaAAP5 (e), BnaAAP6 (f), BnaAAP7 (g) and BnaAAP8 (h) genes under nitrate $\left(\mathrm{NO}_{3}{ }^{-}\right)$and ammonium $\left(\mathrm{NH}_{4}{ }^{+}\right)$conditions. For the transcriptional analysis, the rapeseed seedlings were first hydroponically cultivated under $6.0 \mathrm{mM}$ nitrate $\left(\mathrm{NO}_{3}{ }^{-}\right)$for $10 \mathrm{~d}$, and then were transferred to an $\mathrm{N}$-free solution for $3 \mathrm{~d}$. Subsequently, the above seedlings were sampled after treatment with $6.0 \mathrm{mM}$ ammonium $\left(\mathrm{NH}_{4}^{+}\right)$for $3 \mathrm{~d}$. The shoots and roots were individually sampled, and each sample includes three independent biological replicates. The significance level of $P<0.05$ is used as the threshold to identify the differential BnaAAP expression under high and low nitrate treatments. The differentially expressed genes with higher expression between different treatments in the shoots or roots are indicated with asterisks

possibly involved in phosphate-regulated nitrate response, which indicates the interaction between $\mathrm{N}$ and phosphorus nutrients. Under phosphate limitation condition, a total of 15 BnaAAP DEGs were identified in the shoots or roots (Fig. 9, Additional file 1: Table S2). In the shoots, no differential expression of BnaAAP5s and BnaAAP6s was observed between sufficient phosphate and insufficient phosphate conditions (Fig. 9e, f). However, the DEGs of other BnaAAPs were upregulated by limited phosphate (Fig. 9a-d, g-h). In the roots, 13 


\begin{tabular}{|c|c|c|c|c|c|c|c|c|c|c|c|}
\hline \multirow[t]{2}{*}{ (A) } & \multicolumn{2}{|c|}{ Shoot } & \multicolumn{2}{|c|}{ Root } & \multirow{2}{*}{\multicolumn{2}{|c|}{ (B) }} & \multicolumn{2}{|c|}{ Shoot } & \multicolumn{2}{|c|}{ Root } & \multirow{6}{*}{$\begin{array}{c}0 \\
20 \\
40 \\
80 \\
100\end{array}$} \\
\hline & $+P i$ & $-P i$ & $+\mathrm{Pi}$ & $-P i$ & & & $+\mathrm{Pi}$ & $-\mathrm{Pi}$ & $+\mathrm{Pi}$ & $-\mathrm{Pi}$ & \\
\hline BnaA1.AAP1 & & & & * & \multirow{4}{*}{$\begin{array}{c}0 \\
10 \\
20 \\
30\end{array}$} & $B n a A 3 . A A P 2$ & & * & & \multirow[b]{2}{*}{$*$} & \\
\hline$B n a A 9 . A A P 1$ & & * & & $*$ & & \multirow{2}{*}{$\begin{array}{l}\text { BnaA10.AAP2 } \\
B n a C 3 . A A P 2\end{array}$} & & & & & \\
\hline BnaC4.AAP1 & & $\star$ & & & & & & \multirow[b]{2}{*}{ * } & & \multirow{2}{*}{$*$} & \\
\hline & & & & & & BnaC9.AAP2 & & & & & \\
\hline (C) & \multicolumn{2}{|c|}{ Shoot } & \multicolumn{2}{|c|}{ Root } & \multirow[b]{2}{*}{0} & \multirow[t]{2}{*}{ (D) } & \multicolumn{2}{|c|}{ Shoot } & \multicolumn{2}{|c|}{ Root } & \\
\hline & $+\mathrm{Pi}$ & $-\mathrm{Pi}$ & $+\mathrm{Pi}$ & $-\mathrm{Pi}$ & & & $+\mathrm{Pi}$ & $-\mathrm{Pi}$ & $+\mathrm{Pi}$ & $-\mathrm{Pi}$ & \\
\hline BnaA7.AAP3 & & & * & & 1 & BnaA2.AAP4 & & * & & * & 0 \\
\hline BnaC6.AAP3a & & & * & & $\begin{array}{l}2 \\
3\end{array}$ & BnaA6.AAP4 & & & & * & $\begin{array}{l}2 \\
4\end{array}$ \\
\hline & & & & & & BnaC2.AAP4 & & * & & * & 8 \\
\hline & & & & & & BnaC3.AAP4 & & & & * & \\
\hline (E) & Sh & & Ro & & & (F) & Sh & & Ro & & 0 \\
\hline & $+\mathrm{Pi}$ & $-\mathrm{Pi}$ & $+\mathrm{Pi}$ & $-\mathrm{Pi}$ & & & $+P i$ & $\overline{-P i}$ & $+\mathrm{Pi}$ & $-\mathrm{Pi}$ & 2 \\
\hline BnaA5.AAP5 & & & & & $\begin{array}{c}0 \\
10\end{array}$ & BnaCn.AAP6 & & & & * & $\begin{array}{l}4 \\
6\end{array}$ \\
\hline BnaA8.AAP5 & & & & & $\begin{array}{l}10 \\
20\end{array}$ & & & & & & \\
\hline BnaA10.AAP5 & & & & & 30 & & & & & & \\
\hline BnaC6.AAP5 & & & & & & & & & & & \\
\hline (G) & Sh & & Ro & & & (H) & Sh & & Ro & & \\
\hline & $+P i$ & $-\mathrm{Pi}$ & $+\mathrm{Pi}$ & $\overline{-P i}$ & & & $+\mathrm{Pi}$ & $\overline{-P i}$ & $+\mathrm{Pi}$ & $\overline{-P i}$ & \\
\hline BnaA9.AAP7 & & * & & & $\begin{array}{l}0 \\
1\end{array}$ & BnaC5.AAP8 & & & & & 0 \\
\hline BnaC9.AAP7 & & & & & 2 & BnaC8.AAP8a & & & & * & 6 \\
\hline & & & & & & BnaC8.AAP8b & & * & & * & $\begin{array}{c}8 \\
10\end{array}$ \\
\hline & & & & & & BnaC8.AAP8c & & * & & & \\
\hline $\begin{array}{l}\text { Fig. } 9 \text { The qRT-PCR-assisted trans } \\
\text { (Pi) levels. Differential expression } \\
\text { (h) genes under high }(+, 250 \mu \mathrm{M}) \\
\text { seedlings after seed germination } \\
\text { phosphate for } 3 \mathrm{~d} \text { until sampling. } \\
\text { replicates. The significance level } \\
\text { treatments. The differentially expre } \\
\text { with asterisks }\end{array}$ & $\begin{array}{l}\text { criptic } \\
\text { of the } \\
\text { phos } \\
\text { were } \\
\text { The } \\
\text { f } P< \\
\text { essed }\end{array}$ & $05 \mathrm{i}$ & $\begin{array}{l}\text { aracter } \\
\text { P1 (a), } \\
\text { and lov } \\
\text { dropor } \\
\text { and ro } \\
\text { used a } \\
\text { with r }\end{array}$ & $\begin{array}{l}\text { zat } \\
\text { Bn } \\
\text { (- } \\
\text { ica } \\
\text { ts } \\
\text { th } \\
\text { igh }\end{array}$ & $\begin{array}{l}\text { amine } \\
\text { BnaAA } \\
\text { Dspha } \\
\text { inder } \\
\text { dually } \\
\text { to ic } \\
\text { on be }\end{array}$ & $\begin{array}{l}\text { acid permease } \\
3 \text { (c), BnaAAP4 } \\
\text { levels. For the } \\
50 \mu \mathrm{M} \text { phospha } \\
\text { sampled, and e } \\
\text { ntify the differe } \\
\text { veen different }\end{array}$ & $\begin{array}{l}\text { AAP) } \\
\text { d), } B r \\
\text { trans } \\
\text { te (Kh } \\
\text { ach s } \\
\text { intial } \\
\text { reatm }\end{array}$ & $\begin{array}{l}\text { yenes } \\
\text { aAAPs } \\
\text { criptio } \\
\left.\mathrm{H}_{2} \mathrm{PO}_{4}\right) \\
\text { ample } \\
\text { BnaAA } \\
\text { ents i }\end{array}$ & $\begin{array}{l}\text { n Bras } \\
\text { (e), Br } \\
\text { hal ana } \\
\text { for } 10 \\
\text { includ } \\
\text { expre } \\
\text { the } s\end{array}$ & $\begin{array}{l}\text { ica no } \\
\text { aAAP } \\
\text { lysis, } \\
\text { d, ane } \\
\text { es thre } \\
\text { ssion } \\
\text { hoots }\end{array}$ & $\begin{array}{l}\text { ler different phosphate } \\
\text { AAP7 }(\mathbf{g}) \text { and BnaAAP8 } \\
\text { ld uniform B. napus } \\
\text { ere transferred to } 5 \mu \mathrm{M} \\
\text { endent biological } \\
\text { igh and low phosphate } \\
\text { are indicated }\end{array}$ \\
\hline
\end{tabular}

BnaAAPs exhibited differential expression, however, we did not identify the differential expression of BnaAAP5s and BnaAAP7s between sufficient phosphate and insufficient phosphate conditions (Fig. 9e, g). The DEGs of BnaAAP1s, BnaAAP2s, BnaAAP4s, BnaAAP6s, and $B n a A A P 8 s$ presented higher expression levels under phosphate insufficiency than under phosphate sufficiency condition (Fig. 9a, b, d, f, h). By contrast, insufficient phosphate supply repressed the expression of BnaAAP3s in the roots (Fig. 9c).

Boron is an essential micronutrient for vegetative and reproductive growth in plants [39], and our previous studies reveal that $B$. napus is hypersensitive to boron deficiency [40, 41]. In rapeseed plants, Wang et al. [42] found that boron deprivation enhances protein degradation and influences AA metabolism. Gas chromatography-mass spectrometry (GC-MS)-based metabolomics reveals that AA accumulation is increased in boron-deficient citrus [43]. Under deficient boron condition, we identified a total of 21 BnaAAP DEGs in the shoots and roots (Fig. 10a, c$\mathrm{h}$, Additional file 1: Table S2) whereas we did not detect any differential expression of BnaAAP2s (Fig. 10b, Additional file 1: Table S2). In the shoots, the expression of the BnaAAP1, BnaAAP4, BnaAAP5, BnaAAP6, and BnaAAP8 DEGs was significantly upregulated by boron deficiency whereas the BnaAAP3 and BnaAAP7 DEGs showed the opposite expression patterns (Fig. 10c, g; Additional file 1: Table S2). In the roots, we found that the 
low boron significantly induced the expression of the BnaAAP1, BnaC3.AAP4, and BnaAAP5 DEGs (Fig. 10a, e; Additional file 1: Table S2), whereas repressed the expression of the BnaC2.AAP4 and BnaAAP8 DEGs (Fig. 10d, h; Additional file 1: Table S2).

Cadmium is a non-essential heavy metal that is highly biotoxic for the growth and development of plant species [44]. Rapeseed has great potential for the phytoremediation of cadmium-contaminated soils [45]. Plants exhibit higher concentrations of AAs under cadmium toxicity than under cadmium-free conditions [46]. The increase in AA concentrations under cadmium stresses may be associated with their possible integration into phytochelatins, which serve as metal chelators to alleviate cell damages induced by cadmium toxicity [47, 48]. Under cadmium toxicity, we identified a total of 21 BnaAAP DEGs in the shoots and roots (Fig. 11, Additional file 1: Table S2). In the shoots, the expression of the BnaAAP1, BnaAAP2, BnaAAP3, BnaAAP5, BnaAAP6, and BnaAAP7 DEGs was significantly elevated under cadmium toxicity (Fig. 11a-c, e-g), which repressed the expression of BnaAAP4s and BnaAAP8s (Fig. 11d, h). In

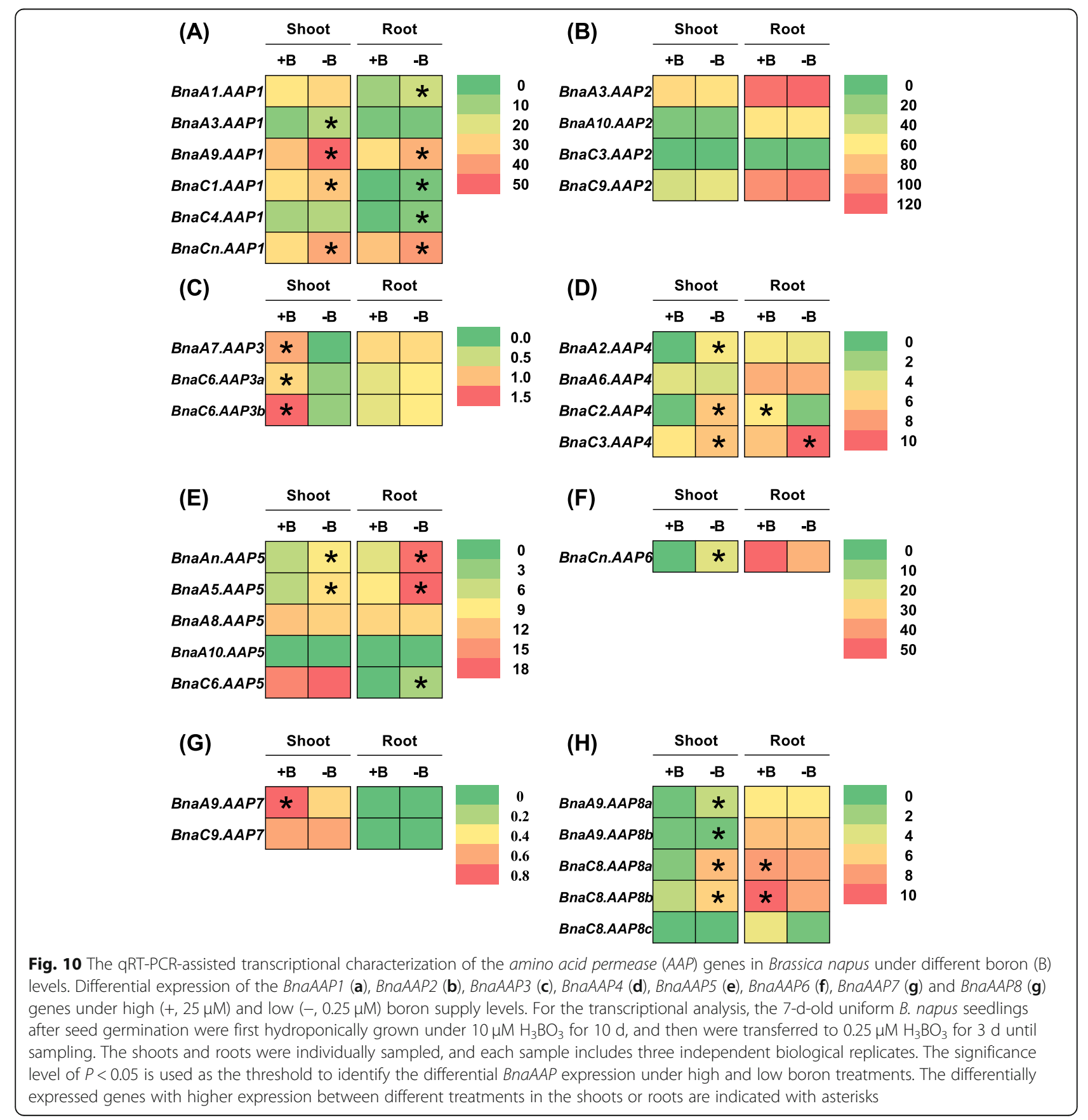


the roots, the expression of most BnaAAPs was significantly upregulated by cadmium toxicity except that the $B n a C n . A A P 1$ expression was obviously downregulated by cadmium toxicity.
Soil salinity is one of the most important environmental factors that constrain plant growth, and development, and salt stress significantly reduces rapeseed yield [49]. Salt stress induces an obvious increase in AA concentrations [50], and the enhanced $A A P$ expression promotes
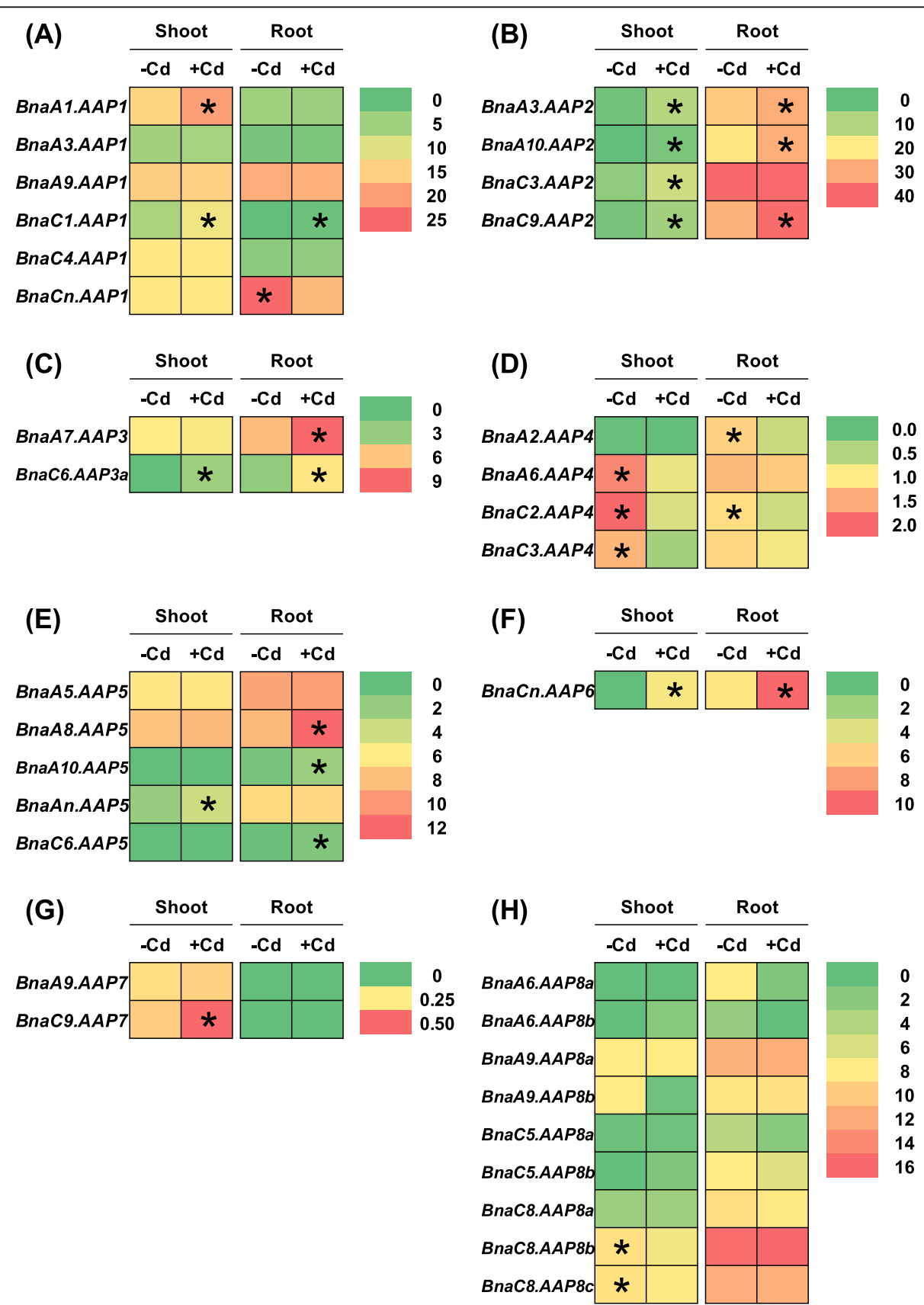

Fig. 11 The qRT-PCR-assisted transcriptional characterization of the amino acid permease (AAP) genes in Brassica napus under cadmium (Cd) toxicity. Differential expression of the BnaAAP1 (a), BnaAAP2 (b), BnaAAP3 (c), BnaAAP4 (d), BnaAAP5 (e), BnaAAP6 (f), BnaAAP7 (g) and BnaAAP8 (h) genes under $\mathrm{Cd}$-free $(-\mathrm{Cd})$ and $\mathrm{Cd}(10 \mu \mathrm{M} \mathrm{CdCl})$ toxicity. For the transcriptional analysis, the 7-d-old uniform $B$. napus seedlings after seed germination were hydroponically cultivated in a cadmium-free solution for $10 \mathrm{~d}$, and then were transferred to $10 \mu \mathrm{M} \mathrm{CdCl}$ for $12 \mathrm{~h}$ until sampling. The shoots and roots were individually sampled, and each sample includes three independent biological replicates. The significance level of $P<0.05$ is used as the threshold to identify the differential BnaAAP expression under cadmium-free and cadmium treatments. The differentially expressed genes with higher expression between different treatments in the shoots or roots are indicated with asterisks 
plant salt tolerance [51]. Under salt stress, we identified a total of $23 A A P$ DEGs in the shoots and roots. In the eight $A A P$ subgroups, we did not detect differential expression of BnaAAP5s (Fig. 12). Most of the DEGs, particularly BnaAAP1s and BnaAAP2s, showed higher expression levels under salt stress than under the nonsalt condition (Fig. 12).

To investigate whether BnaAAPs were responsive to diverse nutrient stresses simultaneously, we constructed a Venn diagram with the identified DEGs. As shown in Fig. 13, BnaC8.AAP8b was simultaneously regulated by low nitrate, excessive ammonium, limited phosphate, deficient boron, toxic cadmium, and salt stress conditions in the shoots (Fig. 13a). This result indicated that Bna$C 8 . A A P 8 b$ might play a core role in regulating rapeseed resistance to nutrient stresses through the modulation of AA transport. Although we did not detect any $A A P$ homolog simultaneously responsive to these six nutrient stresses in the rapeseed roots, we found several $A A P$ homologs, such as BnaA1.AAP1s and BnaA9.AAP1, simultaneously responsive to three or four nutrient stresses (Fig. 13b).

\section{Discussion}

Previous studies have shown that the AAP family members play critical roles in plant growth, organ development, and abiotic and biotic stress responses [4-7]. However, there have been few systematic studies on $A A P s$ in $B$. napus so far. In this study, we identified a total of 34 full-length $A A P$ homologs representing eight subgroups $(A A P 1-A A P 8)$ in the allotetraploid rapeseed genome $\left(\mathrm{A}_{\mathrm{n}} \mathrm{A}_{\mathrm{n}} \mathrm{C}_{\mathrm{n}} \mathrm{C}_{\mathrm{n}}\right)$. We found some homolog number variations of $A A P S$ and analyzed their phylogeny relationships in Brassica species. In addition, we also analyzed the physio-chemical characteristics, gene/protein structures, conserved AA transport motifs, Darwin's evolutionary pressure, and CREs of AAPs in B. napus. Eventually, we delineated the differential expression profile of $B n a A A P$ s under nitrate limitation, ammonium excess, phosphate shortage, boron deficiency, cadmium toxicity, and salt stress. The global landscapes of BnaAAPs will provide an integrated insight into their family evolution and AAP-mediated AA transport.

\section{Homolog number variations of $A A P s$ in allotetraploid rapeseed indicated their functional divergence}

Our previous study has shown that the genes within a family usually exhibit obvious number variations during their evolutionary process [52], which further contributes to gene family division, expansion, and functional divergence [53]. B. napus, which is formed by hybridization of the diploid $B$. rapa and $B$. oleracea, undergoes several rounds of whole-genome triplication and duplication compared with Arabidopsis [24].
Therefore, these processes usually causes the formation of multicopy (four to six, or more) gene family in allotetraploid rapeseed [54]. In this study, we found that the homolog number variation events of $A A P s$ also occurred in allotetraploid rapeseed (Fig. 1b). In the model Arabidopsis, each $A A P$ had a single copy (Fig. 1a), however, the copy number of BnaAAPs varied from one (BnaAAP6) to nine (BnaAAP8s) (Fig. 1b). The result indicated that there might exist the functional divergence of the BnaAAP homologs.

\section{Differential expression profiling of BnaAAPs implied their potential involvement in the responses of rapeseed plants to diverse nutrient stresses}

Environmental stresses lead to AA accumulation in leaves due to the decrease in AA usage for protein biosynthesis or the increase in AA release from protein degradation [55]. The changes in AA composition in the cells in response to environmental stresses results in the expression alterations of AATs [56].

About $75 \%$ of the leaf $\mathrm{N}$ nutrients in the $\mathrm{C}_{3}$ plants are stored in the form of chloroplast proteins [57], which are considered as the main sources for $\mathrm{N}$ reallocation within the plant shoots [58]. Under $\mathrm{N}$ deficiency, these chloroplast proteins in the old or senescent leaves are degraded into amide- $\mathrm{N}$ compounds, particularly AAs, which are reallocated into the newly developing leaves [10]. Therefore, optimal expression of the AAP genes will contribute to the efficient transport of AAs, thus favoring the enhancement of crop $\mathrm{N}$ limitation adaptation and NUE.

A previous study determined the AA concentrations in all subcellular compartments and characterized the $A A P$ family transporters. However, three $A A P$ genes were identified and their expression profiling was characterized at different nitrate levels [59]. In another study, 8 $A A P$ families (AAP1-AAP8) containing $18 A A P$ members were identified in rapeseed, but they does not fully cover the genome-wide $A A P$ homologs [60]. Different from our present study, previous studies focused on the $A A P$ expression patterns during different growth and developmental stages of $B$. napus.

In this study, we found that most of the BnaAAP family genes were upregulated in the shoots or roots under limited nitrate supply (Fig. 7). We speculated that the enhanced expression of BnaAAPs might contribute to AA loading into the vascular tissues for long-distance translocation in the roots, and it might facilitate efficient transport of AAs from source leaves to sink organs in the shoots. Ammonium is a major inorganic $\mathrm{N}$ source for plants. At low external supplies, ammonium promotes plant growth, whereas at high external supplies it causes toxicity [61]. When plants are exposed to ammonium stresses, excessive ammonium can be involved in 


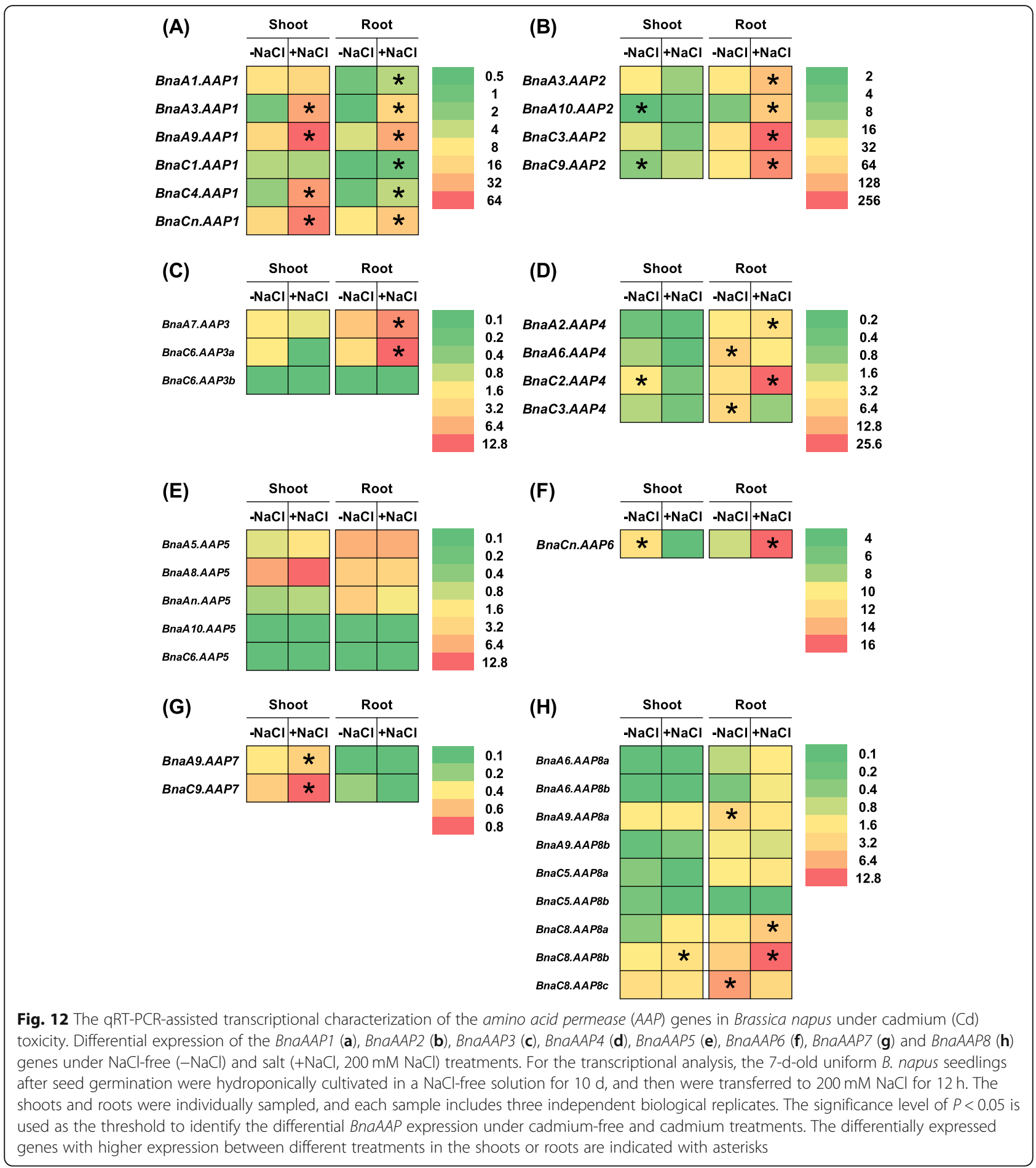

the biosynthesis of AAs mainly through the glutamine synthetase/glutamate synthase pathway, and then surplus ammonium or the newly synthesized AAs are translocated from roots to shoots [62]. In this study, we found that the expressions of some BnaAAPs were altered in response to ammonium toxicity (Fig. 8), which indicated their potential involvement in the alleviation of excessive ammonium-induced damages in rapeseed plants.

In this study, we found that all of the BnaAAP DEGs were upregulated in both the shoots and roots under phosphate deficiency (Fig. 9). It suggested that the phosphate deficiency might facilitate the efficient transport of AA, thus enhancing the adaptation of rapeseed plants to 


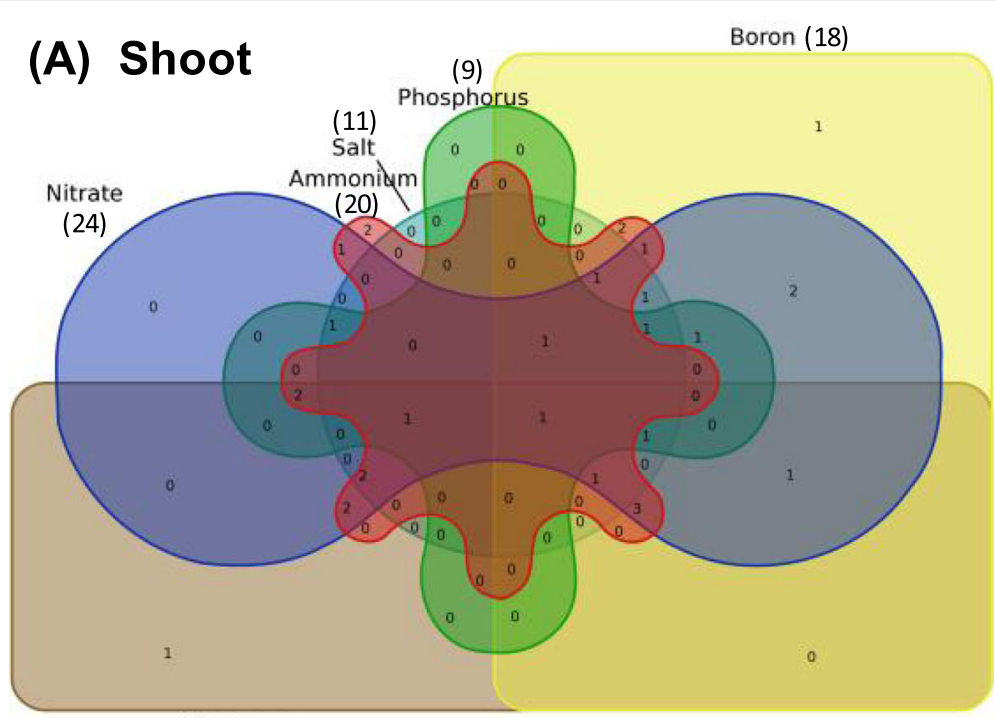

Cadmium (15)

(B) Root

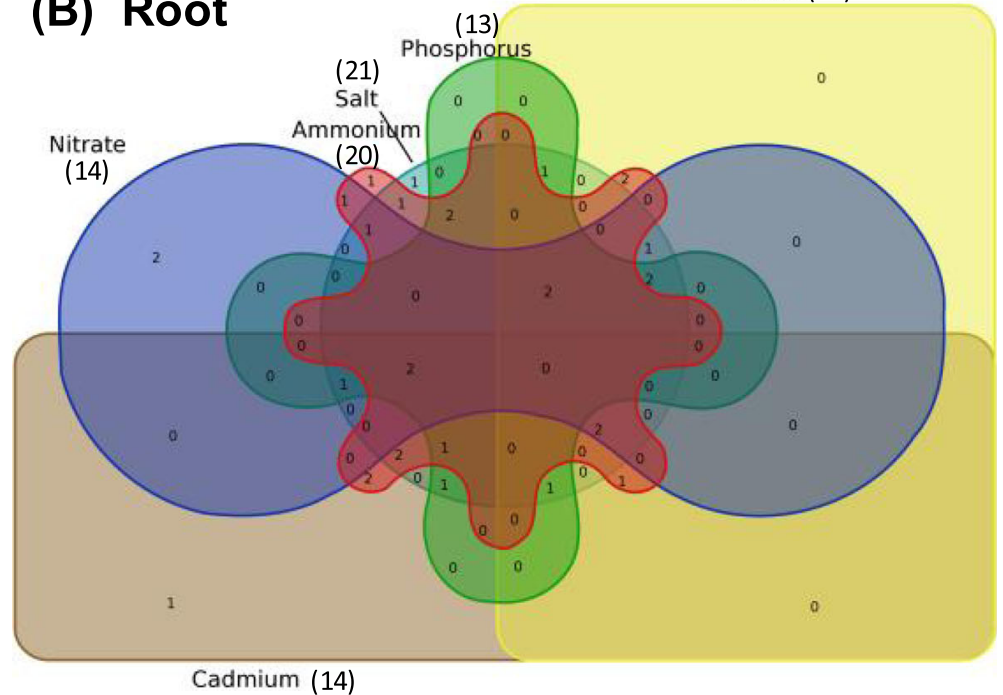

Fig. 13 Venn diagram showing the transcriptional responses of the amino acid permease (AAP) genes in the shoots (a) and roots (b) of Brassica napus under diverse nutrient stresses. The differentially expressed genes between the control and treatments were listed in the brackets

the phosphate-starvation environment. In this study, we found that most of the low boron stress-responsive $B n a A A P s$, such as BnaAAP1s and BnaAAP4-6s, were upregulated (Fig. 10). Therefore, it could be concluded that limited boron supply might trigger AA import and translocation in rapeseed plants by enhancing the $A A T$ expression. The majority of cadmium-responsive BnaAAPs, except $B n a A A P 4 s$ and BnaAAP8s, showed higher expression levels under cadmium toxicity than under the cadmium-free condition (Fig. 11). Based on the aforementioned findings, we proposed that the enhanced expression of BnaAAPs might contribute to efficient AA transport, and it further facilitated the biosynthesis of cadmium-chelators, therefore enhancing plant cadmium resistance. In addition, we also found that salt stress led to a significant increase in the expression of most
BnaAAPs in both the shoots and roots (Fig. 12), and might be associated with salt stress inducing an obvious increase in AA concentrations [50], which contributed to enhancing plant salt resistance [51].

Take together, our above results showed that BnaAAPs were responsive to diverse nutrient stresses, which implied the essential roles of BnaAAPs in the resistance or adaptation of rapeseed plants to stresses.

Elite $A A P s$ could be utilized to enhance crop resistance to biotic and abiotic stresses through genetic engineer Antisense inhibition of StAAP1 expression in potato leaves reduces AA content in potato tubers [63]. The over-expression of PSAAP1 in pea plants contributes to seed AA accumulation and yield enhancement [64]. 
AAP1-mediated increased proline uptake enhances salt tolerance $\mathrm{n}$ Arabidopsis seedlings [51]. Genetic variation in Dip5, an $A A P$ member, regulates glyphosate resistance in Saccharomyces cerevisiae [65]. Knocking out the Arabidopsis AAP2 leads to increased $\mathrm{N}$ allocation to photosynthetically active source leaves, independently of soil $\mathrm{N}$ availability to the plants [15]. The PsAAP6 function in root nodules affects both shoot and root $\mathrm{N}$ supply [6]. All these, the $A A P$ family genes play pivotal roles in crop resistance to diverse stresses.

This study characterized global landscapes of $B n a A A P s$, which will provide a comprehensive understanding of this family gene evolution and AAPmediated AA transport. Our genome-wide identification and molecular characterization of the core BnaAAPs may provide elite gene resources and favor the genetic improvement of rapeseed resistance to nutrient stresses, such as nitrogen limitation, ammonium toxicity, phosphate deficiency, boron deficiency, cadmium toxicity and salt stress, through the molecular modulation of $A A T s$, particularly AAPs.

\section{Methods}

\section{Retrieval of $A A P$ gene sequences}

Using the AA sequences of AAPs from A. thaliana as sources, we conducted a BLASTp search for the AAP homologs in B. rapa, B. oleracea and $B$. napus. In this study, we retrieved the $A A P$ gene sequences using the following databases: The Arabidopsis Information Resource (TAIR10, https://www.arabidopsis.org/) for $A$. thaliana, Brassica Database (BRAD) v. 1.1 (http://brassicadb.org/brad/) for B. rapa [66], Bol base v. 1.0 (http://119.97.203.210/bolbase/index.html) for B. oleracea [67], Genoscope (http://www.genoscope.cns.fr/brassicanapus/) for B. napus [24], National Center for Biotechnology Information (NCBI, www.ncbi.nlm.nih. gov), EnsemblPlants (http://plants.ensembl.org/index. html), and Phytozome v. 10 (http://phytozome.jgi.doe. gov/pz/portal.html) [68].

\section{Gene nomenclature of BnaAAPs}

In this study, based on the nomenclature previously proposed [69-71], we named the AAP genes in Brassica species following the criterion: genus (one capital letter) + plant species (two lowercase letters) + chromosome (followed by a period) + name of the $A A P$ homologs in A. thaliana. For example, BnaA1.AAP1 represents an Arabidopsis AAP1 homolog on the chromosome A1 of B. napus.

\section{Physical mapping and gene expansion analysis of BnaAAPs}

We determined the genomic locations of BnaAAPs by BLASTn search with the complete nucleotide sequences of AtAAPs. Using the genomic annotation, we physically mapped the AtAAPs and BnaAAPs onto the chromosomes using the MapGene2Chromosome v2.1 (http:// mg2c.iask.in/mg2c_v2.1/). In this study, we defined the tandem duplicated genes as an array of two or more $A A P$ genes within a 100-kb genomic region.

\section{Multiple sequence alignment and phylogeny analysis}

We aligned the full-length sequences of the AAP proteins of Arabidopsis and B. napus using ClustalW [72] within MEGA (Molecular Evolutionary Genetics Analysis) v. 6.06 (http://www.megasoftware.net/) [73]. After these alignments, we constructed the phylogenetic trees with the neighbor-joining (NJ) method [74]. We set the Poisson correction, pairwise deletion, and bootstrapping (1000 replicates; random seeds) as the required parameters.

\section{Analysis of evolutionary selection pressure and functional divergence of BnaAAPs}

To determine positive or negative (purifying) selection pressure on BnaAAPs, we calculated the values of Ks, $\mathrm{Ka}$, and $\mathrm{Ka} / \mathrm{Ks}$. First, we performed pairwise alignment of the BnaAAP-AtAAP CDSs using Clustal Omega (http://www.clustal.org/omega/) [75]. Then, we submitted the readout to the KaKs_Calculator (https://sourceforge.net/ projects/kakscalculator2/) software [76] for the calculation of the $\mathrm{Ka}, \mathrm{Ks}$, and $\mathrm{Ka} / \mathrm{Ks}$ with the yn00 method [77]. According to the Darwin's evolution theory, it is proposed that $\mathrm{Ka} / \mathrm{Ks}>$ 1.0 means positive selection, while $\mathrm{Ka} / \mathrm{Ks}<1.0$ indicates purifying selection, and $\mathrm{Ka} / \mathrm{Ks}=1.0$ denotes neutral selection. Further, we calculated the divergence time of BnaAAPs from their progenitors by the following formula: $\mathrm{T}=\mathrm{Ks} / 2 \lambda, \lambda$ $=1.5 \times 10^{-8}$ forBrassicaceaespecies [78].

\section{Molecular characterization of BnaAAPs}

To reveal the molecular characteristics of BnaAAPs, we used the ExPASy ProtoParam (http://www.expasy.org/ tools/protparam.html) [79] program to determine the AA number and composition, MW, pI, GRAVY, and IIs. Values of II $>40.0$ suggest that the proteins are unstable [35]. We used the online WoLF PSORT (http://www. genscript.com/wolf-psort.html) [80] program to predict the subcellular localization. To characterize the transmembrane helices of AtAAPs and BnaAAPs, we submitted their AA sequences to the TMHMM v. 2.0 (http:// www.cbs.dtu.dk/services/TMHMM/) program.

We employed the online SignalP v. 4.1 (http://www. cbs.dtu.dk/services/SignalP/) [81] to predict the presence and location of signal peptide cleavage sites in the AA sequences of BnaAAPs. To determine the recombinant protein solubility, we used the RPSP v. 2009 (http:// 
biotech.ou.edu) program, in which the AAP proteins are assumed to be overexpressed in E. coli [82].

We used the STRING (Search Tool for Recurring Instances of Neighboring Genes) v 11.0 (https://string-db. org) [83] web-server to retrieve and display the repeatedly occurring association networks, including direct (physical) and indirect (function) association, of the AAP proteins in A. thaliana and B. napus.

\section{Conserved motif/domain identification of BnaAAPs}

We used the online InterProScan5 (http://www.ebi.ac. uk/interpro/search/sequence-search) and the conserved domain database (CDD) (http://www.ncbi.nlm.nih.gov/ Structure/bwrpsb/bwrpsb.cgi) to determine the presence of the Aa_trans domain (PAFM01490) of the AAP proteins.

To further examine the structural divergence of the AAP proteins in A. thaliana and Brassica crops, we submitted the protein sequences to the online MEME (Multiple Expectation maximization for Motif Elicitation) $\mathrm{v}$. 4.12.0 (http://meme-suite.org/tools/meme) [84] for the characterization of conserved motifs/domains. We used all the default parameters except the following two parameters: the optimum motif width was set as $6-50 \mathrm{bp}$ and the maximum number of motifs was set as 10 . The conserved motif sequences were presented by the online Weblogo (https://weblogo.berkeley.edu/logo.cgi) [85].

\section{Elucidation of exon-intron structure and putative CREs in promoter regions of BnaAAPs}

Full-length genomic DNA (gDNA) and CDS sequences were collected from the annotated genomes of $A$. thaliana and $B$. napus, and they were used to predict the exon-intron structure of $A A P$ genes. For each $A A P$ gene, a $2.0-\mathrm{kb}$ genomic sequence upstream from the start codon (ATG) was downloaded from the TAIR (https:// www.arabidopsis.org/) website and B. napus Genome Browser (http://www.genoscope.cns.fr/brassicanapus/) [24]. Subsequently, we submitted these sequences to the PLACE v. 30.0 (http://www.dna.affrc.go.jp/PLACE/) program [86] to identify putative CREs.

\section{Transcriptional analysis of BnaAAPs under diverse nutrient stresses}

In this study, the expression patterns of AtAAPs were obtained from the TAIR eFP Browser [87]. To further characterize the transcriptional responses of BnaAAPS under diverse nutrient stresses, we transplanted the 7-dold uniform B. napus seedlings (Zhongshuang 11) after seed germination into black plastic containers with $10 \mathrm{~L}$ Hoagland nutrient solution. The basic nutrition solution contained $1.0 \mathrm{mM} \quad \mathrm{KH}_{2} \mathrm{PO}_{4}, 5.0 \mathrm{mM} \quad \mathrm{KNO}_{3}, 5.0 \mathrm{mM}$ $\mathrm{Ca}\left(\mathrm{NO}_{3}\right)_{2} \cdot 4 \mathrm{H}_{2} \mathrm{O}, \quad 2.0 \mathrm{mM} \quad \mathrm{MgSO}_{4} \cdot 7 \mathrm{H}_{2} \mathrm{O}, \quad 0.050 \mathrm{mM}$ EDTA-Fe, $9.0 \mu \mathrm{M} \mathrm{MnCl}_{2} \cdot 4 \mathrm{H}_{2} \mathrm{O}, 0.80 \mu \mathrm{M} \mathrm{ZnSO} \cdot 7 \mathrm{H}_{2} \mathrm{O}$,
$0.30 \mu \mathrm{M} \quad \mathrm{CuSO}_{4} \cdot 5 \mathrm{H}_{2} \mathrm{O}, 0.10 \mu \mathrm{M} \quad \mathrm{Na}_{2} \mathrm{MoO}_{4} \cdot 2 \mathrm{H}_{2} \mathrm{O}$, and $46 \mu \mathrm{M} \mathrm{H}_{3} \mathrm{BO}_{3}$. The rapeseed seedlings were cultivated in an illuminated chamber following the growth regimes: light intensity of $300-320 \mu \mathrm{mol} \mathrm{m}^{-2} \mathrm{~s}^{-1}$, temperature of $25{ }^{\circ} \mathrm{C}$ daytime $/ 22{ }^{\circ} \mathrm{C}$ night, light period of $16 \mathrm{~h}$ photoperiod/ $8 \mathrm{~h}$ dark, and relative humidity of $70 \%$.

For the nitrate $\left(\mathrm{NO}_{3}{ }^{-}\right)$depletion treatment, the 7-dold uniform $B$. napus seedlings were hydroponically cultivated under high $(6.0 \mathrm{mM})$ nitrate for $10 \mathrm{~d}$, and then were grown under low $(0.30 \mathrm{mM})$ nitrate for $3 \mathrm{~d}$ until sampling. For the ammonium $\left(\mathrm{NH}_{4}^{+}\right)$toxicity treatment, the 7-d-old uniform B. napus seedlings after seed germination were hydroponically cultivated under high nitrate $(6.0 \mathrm{mM})$ for $10 \mathrm{~d}$, and then were grown under $\mathrm{N}$ free condition for $3 \mathrm{~d}$. Finally, the plants were grown under excess ammonium $\left(9.0 \mathrm{Mm} \mathrm{NH}_{4}^{+}\right)$for $6 \mathrm{~h}$ until sampling. For the inorganic phosphate $(\mathrm{Pi})$ starvation treatment, the 7-d-old uniform B. napus seedlings after seed germination were first hydroponically grown under $250 \mu \mathrm{M}$ phosphate $\left(\mathrm{KH}_{2} \mathrm{PO}_{4}\right)$ for $10 \mathrm{~d}$, and then were grown under $5 \mu \mathrm{M}$ phosphate for $3 \mathrm{~d}$ until sampling. For the boron (B) deficiency treatment, the 7-d-old uniform $B$. napus seedlings after seed germination were first hydroponically grown under $10 \mu \mathrm{M} \mathrm{H}_{3} \mathrm{BO}_{3}$ for $10 \mathrm{~d}$, and then were transferred to $0.25 \mu \mathrm{M} \mathrm{H} \mathrm{H}_{3} \mathrm{BO}_{3}$ for $3 \mathrm{~d}$ until sampling. For the cadmium $(\mathrm{Cd})$ toxicity treatment, the 7-d-old uniform B. napus seedlings after seed germination were hydroponically cultivated in a $\mathrm{Cd}$-free solution for $10 \mathrm{~d}$, and then were transferred to $10 \mu \mathrm{M} \mathrm{CdCl}_{2}$ for $12 \mathrm{~h}$ until sampling. For the salt stress treatment, the 7-d-old uniform B. napus seedlings after seed germination were hydroponically cultivated in a $\mathrm{NaCl}$-free solution for $10 \mathrm{~d}$, and then were transferred to $200 \mathrm{mM}$ $\mathrm{NaCl}$ for $1 \mathrm{~d}$ until sampling. The shoots and roots were individually harvested and immediately stored at $-80^{\circ} \mathrm{C}$ until RNA isolation. Each sample contained three independent biological replicates for the transcriptional analyses of BnaAAPs under diverse nutrient stresses.

\section{Quantitative reverse-transcription PCR assays}

The quantitative reverse-transcription polymerase chain reaction (qRT-PCR) assays were used to determine the relative expression of BnaAAPs. After removing genomic DNA from the RNA samples with RNase-free DNase I, complementary DNA (cDNA) synthesis was performed using the PrimeScript ${ }^{\mathrm{m}}$ RT reagent Kit with gDNA Eraser (Perfect Real Time) (TaKaRa, Shiga, Japan) with total RNA as the templates. We performed the quantitative analysis of relative gene expression by using the SYBR $^{\circ}$ Premix Ex Taq ${ }^{\text {tm }}$ II (Tli RNaseH Plus) (TaKaRa, Shiga, Japan) kit in an Applied Biosystems StepOne ${ }^{\text {ts }}$ Plus Real-time PCR System (Thermo Fisher Scientific, Waltham, MA, USA). The thermal recycle regimes were as follows: $95^{\circ} \mathrm{C}$ for $3 \mathrm{~min}$, followed by 40 cycles of $95^{\circ} \mathrm{C}$ 
for $10 \mathrm{~s}$, then $60^{\circ} \mathrm{C}$ for $30 \mathrm{~s}$ [37]. We also conducted a melting curve analysis to ensure the primer specificity of target genes: $95^{\circ} \mathrm{C}$ for $15 \mathrm{~s}, 60^{\circ} \mathrm{C}$ for $1 \mathrm{~min}$, and $60^{\circ} \mathrm{C}-$ $95^{\circ} \mathrm{C}$ for $15 \mathrm{~s}\left(+0.3^{\circ} \mathrm{C}\right.$ per cycle). The public gene BnaEF1- $\alpha$ (forward: GCCTGGTATGGTTGTGACCT; reverse: GAAGTTAGCAGCACCCTTGG) [88] was used as internal references, and the gene expression abundances that were calculated with the slightly modified $2^{-\Delta \Delta C}$ method [89], and the results were showed by heat maps. To ensure the result consistency, we also used a second reference gene BnaGDI1 (forward: GAGTCC CTTGCTCGTTTCC; reverse: TGGCAGTCTCTCCC TCAGAT) [90] to confirm the gene expression consistency. The gene-specific primers for the qRT-PCR assays were listed in the additional file: Table S3.

\section{Statistical analysis}

Statistical analysis was performed using the Student's $t$ test, followed by the Tukey's honestly significant difference (HSD) tests, with the Statistical Productions and Service Solutions (SPSS) 17.0 toolkit. Considering that the qRT-PCR data are normally distributed without logtransformation, we determined the significant difference $(P<0.05)$ in the expression of the target genes using the raw data.

\section{Supplementary information}

Supplementary information accompanies this paper at https://doi.org/10. 1186/s12870-020-02367-7.

Additional file 1: Table S1. Molecular characterization of the amino acid permease (AAP) proteins in Brassica napus. Table S2. Differential expression of each amino acid permease (AAP) gene under diverse nutrient stresses. Table S3. Raw expression data for the amino acid permease (AAP) family genes under diverse nutrient stresses. Figure $\mathbf{S 1}$. Rooted phylogeny analysis of the AAP genes in allotetraploid rapeseed. Figure S2. Trans-membrane characterization of the amino acid permease (AAP) proteins in Arabidopsis thaliana. The TMHMM (http://www.cbs.dtu. $\mathrm{dk} /$ services/TMHMM/) tool was used to predict the transmembrane topology of the AtAAP proteins. Figure S3. Characterization of signal peptides of the amino acid permease (AAP) proteins in Arabidopsis thaliana. The SignalP (http://www.cbs.dtu.dk/services/SignalP/) 4.0 server was used to predict the presence and location of signal peptide cleavage sites in the amino acid sequences of the AtAAP proteins. Figure S4. Tissuespecific expression patterns of the amino acid permease (AAP) genes in Arabidopsis thaliana. (A-H) Relative expression abundances of AtAAP1 (A), $\operatorname{AtAAP2}(\mathrm{B}), \operatorname{AtAAP3}(\mathrm{C}), \operatorname{AtAAP4}(\mathrm{D}), \operatorname{AtAAP5}(\mathrm{E}), \operatorname{AtAAP6}(\mathrm{F}), \operatorname{AtAAP7}(\mathrm{G})$ and AtAAP8 $(H)$ in various tissues. The red and yellow color indicates relative high and low expression levels of AtAAPS.

\footnotetext{
Abbreviations

AA: Amino acid; AAP: Amino acid permease; AAT: Amino acid transporter; At: Arabidopsis thaliana; B: Boron; Bna: Brasssica napus; Bol: Brassica oleracea; Bra: Brassica rapa; BRAD: Brassica Database; Cd: Cadmium; CNV: Copy number variation; CRE: Cis-acting regulatory element; DEGs: Differentially expressed genes; DGE: Differential gene expression; gDNA: Genomic DNA; N: Nitrogen; NCBI: National Center for Biotechnology Information; $\mathrm{NH}_{4}^{+}$: Ammonium; $\mathrm{NO}_{3}^{-}$: Nitrate; NUE: Nitrogen use efficiency; Pi: Phosphate; GRT-PCR: Quantitative reverse-transcription polymerase chain reaction:" TAIR: The Arabidopsis Information Resource;" TF: Transcription factor
}

\section{Acknowledgements}

Not applicable.

\section{Authors' contributions}

HYP, ZT and HJY was involved in data interpretation. CJQ, LY, WWM, and TC cultivated the rapeseed plants, and made the experiments. HJY and HYP designed the study, and ZT and YCP wrote the manuscript. All the authors read and approved the final version of the manuscript.

\section{Funding}

This study was financially supported by the National Natural Science Foundation of China (Grant NO. 31801923). The funder was not involved in the experimental design of the study, data collection, analysis and interpretation, and in writing the manuscript.

\section{Availability of data and materials}

All the data and materials that are required to reproduce these findings can be shared by contacting the corresponding author, Dr. Ying-peng Hua (yingpenghua@zzu.edu.cn).

\section{Ethics approval and consent to participate}

In this study, all the seeds of rapeseed plants were obtained from our research group led by Prof. Jin-yong Huang (jinyhuang@zzu.edu.cn, Zhengzhou University, Zhengzhou, 45000, Henan Province, China).

\section{Consent for publication}

Not applicable.

\section{Competing interests}

The authors declare that they have no competing interests.

\section{Author details}

'School of Agricultural Sciences, Zhengzhou University, Zhengzhou 450000, China. ${ }^{2}$ Sinochem Modern Agricultural Platform, Changchun 130000, China.

Received: 9 November 2019 Accepted: 26 March 2020

Published online: 08 April 2020

\section{References}

1. Zhang GB, Meng S, Gong JM. The expected and unexpected roles of nitrate transporters in plant abiotic stress resistance and their regulation. Int J Mol Sci. 2018:19:3535.

2. Bloom AJ. The increasing importance of distinguishing among plant nitrogen sources. Curr Opin Plant Biol. 2015;25:10-6.

3. Peeters KMU, Vanlaere AJ. Amino-acid metabolism associated with Nmobilization from the flag leaf of wheat (Triticum aestivum L.) during grain development. Plant Cell Environ. 1994;17:131-41.

4. Lu K, Wu B, Wang J, Zhu W, Nie H, Qian J, Huang W, Fang Z. Blocking amino acid transporter OSAAP3 improves grain yield by promoting outgrowth buds and increasing tiller number in rice. Plant Biotechnol J. 2018;16:1710-22.

5. Besnard J, Zhao C, Avice JC, Vitha S, Hyodo A, Pilot G, Okumoto S. Arabidopsis UMAMIT24 and 25 are amino acid exporters involved in seed loading. J Exp Bot. 2018;69:5221-32.

6. Garneau MG, Tan Q, Tegeder M. Function of pea amino acid permease AAP6 in nodule nitrogen metabolism and export, and plant nutrition. J Exp Bot. 2018:69:5205-19.

7. Martho KF, de Melo AT, Takahashi JP, Guerra JM, Santos DC, Purisco SU, Melhem MS, Fazioli RD, Phanord C, Sartorelli P, Vallim MA, Pascon RC. Amino acid permeases and virulence in Cryptococcus neoformans. PLoS One. 2016;11:e0163919.

8. Fischer WN, Loo DD, Koch W, Ludewig U, Boorer KJ, Tegeder M, Rentsch D, Wright EM, Frommer WB. Low and high affinity amino acid $\mathrm{H}^{+}$cotransporters for cellular import of neutral and charged amino acids. Plant J. 2002;29:717-31.

9. Tegeder M, Rentsch D. 2010. Uptake and partitioning of amino acids and peptides. Mol Plant. 2010;3:997-1011.

10. Tegeder M, Masclaux-Daubresse C. Source and sink mechanisms of nitrogen transport and use. New Phytol. 2018;217:35-53. 
11. Okumoto S, Koch W, Tegeder M, Fischer WN, Biehl A, Leister D, Stierhof YD, Frommer WB. Root phloem-specific expression of the plasma membrane amino acid proton co-transporter AAP3. J Exp Bot. 2004;55:2155-68.

12. Tegeder M, Hammes UZ. The way out and in: phloem loading and unloading of amino acids. Curr Opin Plant Biol. 2018;43:16-21.

13. Karmann J, Müller B, Hammes UZ. The long and winding road: transport pathways for amino acids in Arabidopsis seeds. Plant Reprod. 2018;31:25361.

14. Wang J, Wu BW, Lu K, Wei Q, Qian JJ, Chen YP, Fang ZM. The amino acid permease OSAAP5 regulates tiller number and grain yield in rice. Plant Physiol. 2019;180:1031-45.

15. Perchlik M, Tegeder M. Improving plant nitrogen use efficiency through alteration of amino acid transport processes. Plant Physiol. 2018;175:235-47.

16. Svennerstam H, Ganeteg U, Näsholm T. Root uptake of cationic amino acids by Arabidopsis depends on functional expression of amino acid permease. New Phytol. 2008;18:620-30.

17. Hirner B, Fischer WN, Rentsch D, Kwart M, Frommer WB. Developmental control of $\mathrm{H}^{+}$/amino acid permease gene expression during seed development of Arabidopsis. Plant J. 1998;14:535-44.

18. Sanders A, Collier R, Trethewy A, Gould G, Sieker R, Tegeder M. AAP1 regulates import of amino acids into developing Arabidopsis embryos. Plant J. 2009;59:540-52.

19. Schmidt $R$, Stransky $H$, Koch $W$. The amino acid permease AAP8 is important for early seed development in Arabidopsis thaliana. Planta. 2007; 226:805-13.

20. Birnbaum K, Shasha DE, Wang JY, Jung JW, Lambert GM, Galbraith DW, Benfey PN. A gene expression map of the Arabidopsis root. Science. 2003; 302:1956-60.

21. Okumoto S, Schmidt R, Tegeder M, Fischer WN, Rentsch D, Frommer WB, Koch W. High affinity amino acid transporters specifically expressed in xylem parenchyma and developing seeds of Arabidopsis. J Biol Chem. 2002; 277:45338-46.

22. Zhang L, Tan Q, Lee R, Trethewy A, Lee YH, Tegeder M. Altered xylemphloem transfer of amino acids affects metabolism and leads to increased seed yield and oil content in Arabidopsis. Plant Cell. 2010;22:3603-20.

23. Bayer PE, Hurgobin B, Golicz AA, et al. Assembly and comparison of two closely related Brassica napus genomes. Plant Biotechnol J. 2017;15:1602-10.

24. Chalhoub B, Denoeud F, Liu SY, et al. Early allopolyploid evolution in the post-Neolithic Brassica napus oilseed genome. Science. 2014;345:950-3.

25. Sun F, Fan G, Hu Q, Zhou Y, et al. The high-quality genome of Brassica napus cultivar ' $\mathrm{ZS} 11^{\prime}$ ' reveals the introgression history in semi-winter morphotype. Plant J. 2017;92:452-68.

26. Rathke GW, Christen O, Diepenbrock W. Effects of nitrogen source and rate on productivity and quality of winter oilseed rape (Brassica napus L.) grown in different crop rotations. Field Crops Res. 2005:94:103-s.

27. Clément G, Moison M, Soulay F, Reisdorf-Cren M, Masclaux-Daubresse C. Metabolomics of laminae and midvein during leaf senescence and sourcesink metabolite management in Brassica napus L. leaves. J Exp Bot. 2017;69: 891-903.

28. Avice JC, Etienne P. Leaf senescence and nitrogen remobilization efficiency in oilseed rape (Brassica napus L.). J Exp Bot. 2014:65:3813-24.

29. Girondé A, Etienne P, Trouverie J, et al. The contrasting $N$ management of two oilseed rape genotypes reveals the mechanisms of proteolysis associated with leaf $\mathrm{N}$ remobilization and the respective contributions of leaves and stems to $\mathrm{N}$ storage and remobilization during seed filling. BMC Plant Biol. 2015;15:59.

30. Xu G, Guo C, Shan H, Kong H. Divergence of duplicate genes in exon-intron structure. Proc Natl Acad Sci U S A. 2012;109:1187-92.

31. Schmidt R, Acarkan A, Boivin K. Comparative structural genomics in the Brassicaceae family. Plant Physiol Bioch. 2001;39:253-62

32. Schranz ME, Lysak MA, Mitchell-Olds T. The ABC's of comparative genomics in the Brassicaceae: building blocks of crucifer genomes. Trends Plant Sci. 2006;11:535-42

33. Yang $Y$, Lai K, Tai $P$, Li W. Rates of nucleotide substitution in angiosperm mitochondrial DNA sequences and dates of divergence between Brassica and other angiosperm lineages. J Mol Evol. 1999;48:597-604.

34. Su YH, Frommer WB, Ludewig U. Molecular and functional characterization of a family of amino acid transporters from Arabidopsis. Plant Physiol. 2004; 136:3104-13.

35. Guruprasad K, Reddy BV, Pandit MW. Correlation between stability of a protein and its dipeptide composition: a novel approach for predicting in vivo stability of a protein from its primary sequence. Protein Eng. 1990;4: 155-61.

36. Wittkopp PJ, Kalay G. Cis-regulatory elements: molecular mechanisms and evolutionary processes underlying divergence. Nat Rev Genet. 2011:6:59-69.

37. Sheflin AM, Chiniquy D, Yuan C, Goren E, Kumar I, Braud M, Brutnell T, Eveland AL, Tringe S, Liu P, Kresovich S, Marsh EL, Schachtman DP, Prenni JE. Metabolomics of sorghum roots during nitrogen stress reveals compromised metabolic capacity for salicylic acid biosynthesis. Plant Direct. 2019;3:e00122.

38. Hu B, Jiang Z, Wang W, et al. Nitrate-NRT1.1B-SPX4 cascade integrates nitrogen and phosphorus signalling networks in plants. Nat Plants. 2019;5:637.

39. Loomis WD, Durst RW. 1992. Chemistry and biology of boron. BioFactors. 1992:3:229-39.

40. Hua Y, Zhou T, Ding G, Yang Q, Shi L, Xu F. Physiological, genomic and transcriptional diversity in responses to boron deficiency in rapeseed genotypes. J Exp Bot. 2016a;67:5769-84.

41. Hua Y, Zhang D, Zhou T, He M, Ding G, Shi L, Xu F. Transcriptomics-assisted quantitative trait locus fine mapping for the rapid identification of a nodulin 26-like intrinsic protein gene regulating boron efficiency in allotetraploid rapeseed. Plant Cell Environ. 2016b;39:1601-18.

42. Wang Z, Wang Z, Shi L, Wang L, Xu F. Proteomic alterations of Brassica napus root in response to boron deficiency. Plant Mol Biol. 2010;74:265-78.

43. Dong X, Liu G, Wu X, et al. Different metabolite profile and metabolic pathway with leaves and roots in response to boron deficiency at the initial stage of citrus rootstock growth. Plant Physiol Bioch. 2016;108:121-31.

44. Zhang $H$, Reynolds M. Cadmium exposure in living organisms: a short review. Sci Total Environ. 2019;678:761-7.

45. Zhang ZH, Zhou T, Tang TJ, Song HX, Guan CY, Huang JY, Hua YP. A multiomics approach reveals the pivotal role of subcellular reallocation in determining rapeseed resistance to cadmium toxicity. J Exp Bot. 2019;70: 5437-55.

46. Chia MA, Lombardi AT, da Graça Gama Melão M, Parrish CC. Combined nitrogen limitation and cadmium stress stimulate total carbohydrates, lipids, protein and amino acid accumulation in Chlorella vulgaris (Trebouxiophyceae). Aquat Toxicol. 2015;160:87-95.

47. Kumar M, Kumari P, Gupta V, Anisha PA, Reddy CRK, Jha B. Differential responses to cadmium induced oxidative stress in marine macroalga Ulva lactuca (Ulvales, Chlorophyta). Biometals. 2010;23:315-25.

48. Kovacik J, Klejdus B, Hedbavny J, Backor M. Effect of copper and salicyclic acid on phenolic metabolites and free amino acids in Scenedesmus quadricauda (Chlorophyceae). Plant Sci. 2010;178:307-11.

49. Shokri-Gharelo R, Noparvar PM. Molecular response of canola to salt stress: insights on tolerance mechanisms. Peer J. 2018:6:e4822.

50. Xie $Y$, Sun $X$, Feng $Q$, Luo H, Wassie M, Amee M, Amombo E, Chen L. Comparative physiological and metabolomic analyses reveal mechanisms of Aspergillus aculeatus-mediated abiotic stress tolerance in tall fescue. Plant Physiol Biochem. 2019;142:342-50.

51. Wang $T$, Chen $Y$, Zhang M, Chen J, Liu J, Han H, Hua X. Arabidopsis AMINO ACID PERMEASE1 contributes to salt stress-induced proline uptake from exogenous sources. Front Plant Sci. 2017:8:2182.

52. Hua YP, Zhou T, Song HX, Guan CY, Zhang ZH. Integrated genomic and transcriptomic insights into the two-component high-affinity nitrate transporters in allotetraploid rapeseed. Plant Soil. 2018:427:245-68.

53. Ohno S. Gene duplication and the uniqueness of vertebrate genomes circa 1970-1999. Semin Cell Dev Biol. 1999;10:517-22

54. Parkin IA, Gulden SM, Sharpe AG, Lukens L, Trick M, Osborn TC, Lydiate DJ. Segmental structure of the Brassica napus genome based on comparative analysis with Arabidopsis thaliana. Genetics. 2005;171:765-81.

55. Verbruggen $N$, Hermans C. Proline accumulation in plants: a review. Amino Acids. 2008:35:752-9.

56. Wan Y, King R, Mitchell RAC, Hassani-Pak K, Hawkesford MJ. Spatiotemporal expression patterns of wheat amino acid transporters reveal their putative roles in nitrogen transport and responses to abiotic stress. Sci Rep. 2017;7:5461.

57. Evans JR, Clarke VC. The nitrogen cost of photosynthesis. J Exp Bot. 2019;70: 7-15

58. Liu W, Sun Q, Wang K, Du Q, Li WX. Nitrogen Limitation Adaptation (NLA) is involved in source-to-sink remobilization of nitrate by mediating the degradation of NRT1.7 in Arabidopsis. New Phytol. 2017;214:734-44.

59. Tilsner J, Kassner N, Struck C, Lohaus G. Amino acid contents and transport in oilseed rape (Brassica napus L.) under different nitrogen conditions. Planta. 2005;221:328-38. 
60. Song J, Jiang $L$, Jameson PE. Expression patterns of Brassica napus genes implicate IPT, CKX, sucrose transporter, cell wall invertase, and amino acid permease gene family members in leaf, flower, silique, and seed development. J Exp Bot. 2015:66:5067-82.

61. Liu Y, von Wirén N. Ammonium as a signal for physiological and morphological responses in plants. J Exp Bot. 2017:68:2581-92.

62. Vega-Mas I, Cukier C, Coleto I, González-Murua C, Limami AM, GonzálezMoro MB, Marino D. Isotopic labelling reveals the efficient adaptation of wheat root TCA cycle flux modes to match carbon demand under ammonium nutrition. Sci Rep. 2019:9:8925

63. Koch W, Kwart M, Laubner M, Heineke D, Stransky H, Frommer WB, Tegeder $M$. Reduced amino acid content in transgenic potato tubers due to antisense inhibition of the leaf $\mathrm{H}^{+}$/amino acid symporter StAAP1. Plant J. 2003;33:211-20

64. Zhang L, Garneau MG, Majumdar R, Grant J, Tegeder M. Improvement of pea biomass and seed productivity by simultaneous increase of phloem and embryo loading with amino acids. Plant J. 2015;81:134-46.

65. Rong-Mullins X, Ravishankar A, McNeal KA, Lonergan ZR, Biega AC, Creamer JP, Gallagher JEG. Genetic variation in Dip5, an amino acid permease, and Pdr5, a multiple drug transporter, regulates glyphosate resistance in $S$. cerevisiae. PLoS One. 2017:12:e0187522.

66. Wang X, Wu J, Liang J, Cheng F, Wang X. Brassica database (BRAD) version 2.0: integrating and mining Brassicaceae species genomic resources. Database. 2015;2015:1-8

67. Yu J, Zhao M, Wang X, Tong C, Huang S, Tehrim S, Liu Y, Hua W, Liu S. Bolbase: a comprehensive genomics database for Brassica oleracea. BMC Genomics. 2013;14:664.

68. Goodstein DM, Shu S, Howson R, Neupane R, Hayes RD, Fazo J, Mitros T Dirks W, Hellsten U, Putnam N. Phytozome: a comparative platform for green plant genomics. Nucleic Acids Res. 2012;40:D1178-86.

69. Ostergaard L, King GJ. Standardized gene nomenclature for the Brassica genus. Plant Methods. 2008:4:10.

70. Li S, Chen L, Zhang L, Li X, Liu Y, Wu Z, Dong F, Wan L, Liu K, Hong D, Yang G. BnaC9.SMG7b functions as a positive regulator of the number of seeds per silique in Brassica napus by regulating the formation of functional female gametophytes. Plant Physiol. 2015;169:2744-60.

71. Li H, Li J, Song J, Zhao B, Guo C, Wang B, Zhang Q, Wang J, King GJ, Liu K. An auxin signaling gene BnaA3.IAA7 contributes to improved plant architecture and yield heterosis in rapeseed. New Phytol. 2019;222:837-51.

72. Larkin MA, Blackshields G, Brown NP, Chenna R, McGettigan PA, McWilliam $\mathrm{H}$, Valentin F, Wallace IM, Wilm A, Lopez R, Thompson JD, Gibson TJ, Higgins DG. Clustal W and Clustal X version 2.0. Bioinformatics. 2007;23: 2947-8

73. Tamura K, Stecher G, Peterson D, Filipski A, Kumar S. MEGA6: molecular evolutionary genetics analysis version 6.0. Mol Biol Evol. 2013;30:2725-9.

74. Saitou N, Nei M. The neighbor-joining method: a new method for reconstructing phylogenetic trees. Mol Biol Evol. 1987;4:406-25.

75. Sievers F, Wilm A, Dineen DG, et al. Fast, scalable generation of high-quality protein multiple sequence alignments using Clustal omega. Mol Syst Biol. 2011;7:539.

76. Wang DP, Zhang YB, Zhang Z, Zhu J, Yu J. KaKs_Calculator 2.0: a toolkit incorporating gamma-series methods and sliding window strategies. Genomics Proteomics Bioinformatics. 2010:8:77-80.

77. Yang Z, Nielsen R. Estimating synonymous and nonsynonymous substitution rates under realistic evolutionary models. Mol Biol Evol. 2000;17: $32-43$.

78. Blanc G, Wolfe KH. Widespread paleopolyploidy in model plant species inferred from age distributions of duplicate genes. Plant Cell. 2004;16:166778.

79. Gasteiger E, Hoogland C, Gattiker A, Duvaud S, Wilkins MR, Appel RD, Bairoch A. Protein identification and analysis tools on the ExPASy server. In: Walker JM, editor. The Proteomics Protocols Handbook, Humana Press; 2005. p. 571-607.

80. Hamburger D, Horton P, Park KJ, Obayashi T, Fujita N, Harada H. AdamsCollier C J (2007) WoLF PSORT: protein localization predictor. Nucleic Acids Res. 2007;35:W585-7.

81. Petersen TN, Brunak S, von Heijne G, Nielsen H. SignalP 4.0: discriminating signal peptides from transmembrane regions. Nat Methods. 2011;8:785-6.

82. Harrison RG, Bagajewicz MJ. Predicting the solubility of recombinant proteins in Escherichia coli. Methods Mol Biol. 2015:1258:403-8.
83. Szklarczyk D, Gable AL, Lyon D, Junge A, Wyder S, Huerta-Cepas J, Simonovic $M$, Doncheva NT, Morris $J H$, Bork $P$, Jensen $L$, von Mering C. STRING V11: protein-protein association networks with increased coverage, supporting functional discovery in genome-wide experimental datasets. Nucleic Acids Res. 2019;47:D607-13.

84. Bailey TL, Boden M, Buske FA, Frith M, Grant CE, Clementi L, Ren J, Li WW, Noble WS. MEME SUITE: tools for motif discovery and searching. Nucleic Acids Res. 2009;37:W202-8.

85. Crooks GE, Hon G, Chandonia JM, Brenner SE. WebLogo: A sequence logo generator. Genome Res. 2004;14:1188-90.

86. Higo K, Ugawa Y, Iwamoto M, Korenaga T. Plant cis-acting regulatory DNA elements (PLACE) database: 1999. Nucleic Acids Res. 1999;27:297-300.

87. Winter D, Vinegar B, Nahal H, Ammar R, Wilson GV. Provart NJ. PLoS One. 2007;2:e718.

88. Maillard AP, Etienne S, Diquélou J, Trouverie V, Billard V, Yvin JC, Ourry A. Nutrient deficiencies in Brassica napus modify the ionomic composition of plant tissues: a focus on cross-talk between molybdenum and other nutrients. J Exp Bot. 2016;67:5631-41

89. Livak KJ, Schmittgen TD. Analysis of relative gene expression data using real-time quantitative PCR and the $2^{-\Delta \Delta C}$ method. Methods. 2001;25:402-8,

90. Yang HL, Liu J, Huang SM, Guo TT, Deng LB, et al. Selection and evaluation of novel reference genes for quantitative reverse transcription PCR (qRTP(R) based on genome and transcriptome data in Brassica napus L. Gene. 2014;538:113-22.

\section{Publisher's Note}

Springer Nature remains neutral with regard to jurisdictional claims in published maps and institutional affiliations.
Ready to submit your research? Choose BMC and benefit from:

- fast, convenient online submission

- thorough peer review by experienced researchers in your field

- rapid publication on acceptance

- support for research data, including large and complex data types

- gold Open Access which fosters wider collaboration and increased citations

- maximum visibility for your research: over $100 \mathrm{M}$ website views per year

At $\mathrm{BMC}$, research is always in progress.

Learn more biomedcentral.com/submissions 